\title{
Generalized Binary Interaction Parameters for the Peng-Robinson Equation of State
}

Agelia M. Abudour ${ }^{1}$

Sayeed A. Mohammad

Robert L. Robinson, Jr. ${ }^{1}$

${ }^{*}$ Khaled A. M. Gasem ${ }^{1,2}$

\author{
'Oklahoma State University \\ School of Chemical Engineering \\ Stillwater, OK 74078 \\ ${ }^{2}$ University of Wyoming \\ Department of Chemical \& Petroleum Engineering \\ Laramie, WY 82071
}

*Author to whom all correspondence should be sent:

Email: gasem@okstate.edu

Submitted to Fluid Phase Equilibria 


\section{Abstract}

Accurate predictions of phase equilibria of mixtures are essential for designing and optimizing separation processes and numerous other unit operations in the chemical industry. Although experiments provide accurate data, they are time consuming and expensive. A need exists for accurate, reliable models that can provide predictions of vapor-liquid equilibrium (VLE) of mixtures without the need for extensive experimentation. Cubic equations of state (CEOS), such as Soave-Redlich-Kwong (SRK) and Peng-Robinson (PR), are widely used in the chemical industry to perform reservoir simulations and process design calculations due to their inherent simplicity and efficiency. These equations have one or two empirical binary interaction parameters (BIPs) for each binary pair of a mixture. The BIPs cannot be predicted a priori from existing theory, but they can be regressed from VLE experimental data. The present study focused on developing reliable generalized models to estimate the BIPs for the PR EOS using a quantitative structure-property relationship (QSPR) modeling approach.

For model development, a VLE database consisting of 916 low-pressure binary systems covering a wide range of functional-group interactions was utilized. Using this database, the BIPs for the PR EOS were determined by regressing the VLE experimental data. Then, the BIPs were generalized using QSPR modeling methodology. Results indicate that the generalized PR-QSPR model provides bubblepoint pressure predictions within two times the errors obtained through direct regressions of the BIPs. Further, the generalized model was validated by predicting bubble-point pressures of binary systems not used in model development and systems 
that contained data at higher pressures. For 22 systems used for model validation, the developed PR-QSPR model provided generalized predictions comparable to those of data used for model development.

Keywords: Peng-Robinson, cubic equation of state, vapor-liquid equilibria, generalized model, binary interaction parameters, QSPR modeling, neural networks 


\section{Introduction}

Accurate vapor-liquid equilibrium (VLE) predictions are essential for designing and modeling chemical processes. Such equilibrium systems can involve a variety of different molecules, types of interactions and phase conditions. Consequently, they produce different types of phase behavior. The complexity of the phase behavior encountered necessitates different computational frameworks. Although experiments provide accurate data at specific phase conditions, such data are limited and cannot be expected to meet the ever-expanding industrial needs for process design and development [1]. Therefore, a need exists for models that can provide accurate predictions of vapor-liquid equilibrium for a wide variety of systems exhibiting varied phase behaviors.

Two approaches are commonly used in describing VLE behavior of systems. The first approach utilizes an equation of state (EOS) to describe the phase behavior for both the liquid and vapor phases. The second approach utilizes an activity coefficient model to describe the liquid phase while a different model, usually an equation of state, is used to describe the vapor phase. The EOS model has been successful in describing systems containing nonpolar and slightly polar components over broad ranges of temperature and pressure. In contrast, activity coefficient models have been preferred to describe highly non-ideal systems. The activity coefficient model approach has been applied successfully at low temperatures where the liquid phase is relatively incompressible and up to moderate pressures [2]. However, this model is deficient in describing phase behavior at higher pressures. Further, an activity coefficient model is 
incapable of describing the phase behavior in the near-critical region of a mixture. An equation of state model is capable of reasonable predictions of phase behavior in nearcritical region [3]. Additional problems result when the activity coefficient model is used for supercritical components [4]. Therefore, an EOS model applicable to both liquid and vapor phases over a wide range of temperatures and pressures provides several advantages relative to the activity coefficient approach. For the above reasons, an EOS model was used in this work to investigate the model's capability to predict phase behavior of highly non-ideal systems.

Cubic equations of state (CEOS) such as Soave-Redlich-Kwong (SRK) [5] and Peng-Robinson (PR) [6] are widely used in the chemical industry to perform reservoir simulations and process design calculations due to their inherent simplicity and efficiency. The accurate description of phase equilibrium from a CEOS often requires adjustable parameters in the mixing rules, which are referred to as the binary interaction parameters (BIPs). The BIPs account for the unlike molecular interactions in mixtures composed of widely differing molecular species. In fact, an accurate description of phase equilibria of mixtures is generally sensitive to the mixing rules and these parameters in the CEOS [7].

Several mixing rules for EOS parameters have been proposed [8-14]. Nevertheless, the one-fluid mixing rules are simple and relatively accurate for many systems. The CEOS with these mixing rules can be utilized to represent several systems within the experimental precision in many applications [10]. While the use of one interaction parameter is sufficient for some binary systems, other systems, especially those exhibiting polarity and dissimilarity in molecular sizes, require two 
binary parameters. Although these simple mixing rules are not as accurate as theoretically-based mixing rules such as Wong-Sandler mixing rules[14] in representing asymmetric mixtures [15], their simplicity continues to make them attractive, especially when binary interaction parameters (BIPs) are utilized. Therefore, we elected to use the classical one-fluid mixing rules in this study for describing the vapor-liquid equilibria of binary systems.

The classical one-fluid mixing rules require binary interaction parameters $\left(C_{i j}, D_{i j}\right)$ that account for deviations from simple mixing rules. These parameters, in general, cannot be predicted a priori from existing theory, but they can be regressed from experimental measurements on the binary pairs that form the systems of interest. These parameters can have a dramatic effect on the predicted properties of systems and are often required for accurate predictions. Therefore, the need exists for developing reliable generalized models to estimate EOS BIPs.

Several attempts have been made to generalize the binary parameters for a variety of systems. In our previous work [16], we developed generalized correlations for predicting the high-pressure phase equilibria of systems of coalbed gases (methane, nitrogen and carbon dioxide) with water. Several parameter generalizations of varying complexity have been presented for asymmetric mixtures [17-19]. In particular, Gao et al [19] developed generalized-parameter correlations for the conformal combining parameters $\left(\mathrm{N}_{\mathrm{ij}}\right)$ of the PR EOS for asymmetric binary mixtures involving methane, ethane, nitrogen, hydrogen, carbon monoxide and carbon dioxide in the $n$-paraffins $\left(\mathrm{C}_{4}-\right.$ $\mathrm{C}_{44}$ ). A group contribution method has been developed for estimating the temperature dependent binary interaction parameters for mixtures containing alkanes, aromatics and 
naphthenes [20-22]. This model is limited by the relatively small number of group interaction parameters that are available. Lashkarbolooki et al. [23] developed an artificial neural network model for prediction of phase equilibria of binary systems containing carbon dioxide and hydrocarbon systems.

Although several models for generalizing the binary parameters have been proposed in the literature, the existing models suffer from a limited range of applicability and poor generalization when applied to systems for which no data exist. Thus, a generalized model to estimate the interaction parameters of binary systems a priori is required. An accurate generalized model can be obtained when the property models account for molecular structural variations observed in fluid mixtures. One approach that has gained importance is the use of quantitative structure-property relationship (QSPR) and artificial neural networks (ANNs) models to correlate the desired property in terms of molecular structure. Several QSPR models have been proposed in the literature to predict varied and often complex pure-fluid physical properties of molecules [24-28]. However, little work has been done to date on QSPR models for mixtures. Shacham et al. [29] developed a QSPR model for prediction of the binary interaction parameters $\left(C_{i j}\right)$ for cubic equation of state. However, their model was limited to specific homologous series. In contrast, our current study focuses on developing reliable, generalized model applicable for much more diverse range of mixtures. To our knowledge, generalized models to estimate the BIPS of PR EOS for diverse and a large number of binary systems over wide ranges of pressure and temperature have not been presented previously. In this work, the BIPs of the PR EOS were generalized using QSPR 
methodology as outlined in a later section. Thus, a structure-based model capable of $a$ priori prediction of phase behavior for about 1000 binary systems was developed.

This work aims to (a) assess the correlative capabilities of the PR EOS model of describing the phase equilibria of the systems studied using regressed BIPs $\left(C_{i j}\right.$ and $\left.D_{i j}\right)$ in the one-fluid mixing rules (b) develop a QSPR model that can estimate a priori the binary interaction parameters of the PR EOS for a wide variety of systems and (c) evaluate the model predictions using an external test set that was not used in the model development.

The remaining sections are organized as follows: Section 2 presents the PR EOS model and the mixing rules used in this study, Section 3 presents details of the QSPR methodology used to generalize the model parameters, Section 4 discusses the results obtained for correlating and predicting vapor-liquid equilibria for low and high-pressure systems and Section 5 presents the conclusions from this study.

\section{Peng-Robinson Equation of State}

The PR EOS [6] is given as

$$
p=\frac{R T}{v-b}-\frac{a(T)}{v(v+b)+b(v-b)}
$$

where

$$
\begin{aligned}
& a(T)=\frac{0.457535 \alpha(T) R^{2} T_{c}^{2}}{p_{c}^{2}} \\
& b=\frac{0.077796 R T_{c}}{p_{c}}
\end{aligned}
$$


where $p$ is the pressure, $T$ is the temperature, $v$ is the molar volume, $a$ and $b$ are EOS parameters, $T_{c}$ is the critical temperature, $P_{c}$ is the critical pressure and $R$ is the universal gas constant.

The $\alpha(T)$ term in Equation (2) was calculated with the following expression developed in an earlier work [30]:

$$
\alpha(T)=\exp \left(\left(A+B T_{r}\right)\left(1-T_{r}^{C+D \omega+E \omega^{2}}\right)\right)
$$

where $\omega$ is the acentric factor, $T_{r}$ is the reduced temperature and $A$ through $E$ are correlation parameters with values of $2.0,0.836,0.134,0.508$ and -0.0467 , respectively.

\subsection{Mixing Rules}

To apply the PR EOS to mixtures, mixing rules are used to calculate the values of $a$ and b parameters. The classical one-fluid mixing rules were used and are given as [31]

$$
\begin{aligned}
& a=\sum_{i} \sum_{j} z_{i} z_{j} a_{i j} \\
& b=\sum_{i} \sum_{j} z_{i} z_{j} b_{i j} \\
& a_{i j}=\sqrt{a_{i} a_{j}}\left(1-C_{i j}\right) \\
& b_{i j}=\frac{\left(b_{i}+b_{j}\right)}{2}\left(1+D_{i j}\right)
\end{aligned}
$$

where $z$ denotes the molar fraction in the liquid or the vapor phase. In Equations (7) and (8), $C_{i j}$ and $D_{i j}$ are adjustable binary interaction parameters (BIPs) that are regressed from experimental vapor-liquid equilibria data. When $i$ equals $j, C_{i j}$ and $D_{i j}$ values are zero. The most widely-used method is to use a single interaction parameter, $\mathrm{C}_{\mathrm{ij}}$, and frequently it is sufficient for the purpose. The BIPs, $C_{i j}$ and $D_{i j}$, have values typically near zero for systems of non-polar compounds of similar molecular size. However, non-zero 
values of $C_{i j}$ and $D_{i j}$ are generally required in the phase-equilibrium calculations for systems consisting of compounds with large differences in molecular size and shape and for systems containing polar compounds such as water [32]. Although several other mixing rules have been proposed [8-14], the classical one-fluid mixing rules are used more frequently in EOS applications due to their simplicity and efficiency.

\section{QSPR Methodology}

The development of a QSPR model for BIPs of the PR EOS involves several distinct steps which include: (a) database development, (b) PR EOS binary interaction parameter regression, (c) molecular structure generation and optimization, (d) descriptor generation, (e) descriptor reduction, (f) QSPR model development and (g) QSPR model validation. The details of each of these steps have been provided in our previous works [33]. However, for the sake of completeness, a summary of each of each step is outlined below.

Figure 1 presents a flowchart outlining the steps involved in QSPR model development. The modeling process begins by compiling a representative binary VLE database. The binary interaction parameters of the PR EOS are then regressed for each binary system. Then, two-dimensional (2-D) molecular structures of components in each binary system are generated and optimized to find the three-dimensional (3-D) conformation with the least energy. These optimized molecular structures are used to calculate molecular descriptors for each component by using DRAGON [34] and CODESSA [35]. In particular, the current DRAGON [34] software is capable of generating about 4,800 descriptors for each component. This large number of 
generated molecular descriptors is reduced to find the most significant descriptors for predicting the BIPs. These descriptors are then used to develop an artificial neural network (ANN) model. A detail description for each of these different steps follows.

\subsection{Database Development}

Experimental data are essential for developing a reliable phase behavior model. Further, the predictive capability of a QSPR model depends strongly on the accuracy of the experimental data used in the model development process. An experimental VLE database was compiled from available sources covering a sufficient various functional groups and, thus, the compiled database is well suited to test the efficacy of our approach. An earlier study [36] provides additional details of the VLE database used in this study.

Our VLE database consists of a total of 916 binary systems formed from various combinations of 140 different compounds totaling over 33,000 data points (Oklahoma State University, OSU-VLE Database-III) [36]. The experimental VLE data were taken from DECHEMA [37] and NIST-TDE [38]. A majority of the assembled data were lowpressure binary VLE systems. Out of a total of 916 systems, the data for 856 systems were low-pressure binary VLE data (about $0.0005-6$ bar) and the remaining 60 systems contained high-pressure binary VLE data (about 1-58 bar) where the highest pressure is near the critical pressure of the system studied. The range of temperatures for the database used was about 182-554 K. Details of this binary VLE experimental database, along with the temperature, pressure and composition ranges for each binary system, can be found in Table S1 of the Supplementary Material available with the web edition of this work. 
The values of the pure-fluid physical properties, including the critical properties (critical temperature $\left(T_{c}\right)$, critical pressure $\left(P_{c}\right)$ ) and acentric factor $(\omega)$ for each component, constitute the necessary model input variables for the PR EOS. The values of the input variables for the pure components were taken from DIPPR [39].

Figure 2 illustrates the VLE data distribution of the binary systems in the OSU database-III based on chemical classes. The compounds were classified in a similar manner as the UNIFAC functional group classification approach [40]. The database is composed of compounds belonging to 31 chemical classes. Figure 2 shows the number of available binary systems for each type of functional-group interaction. As shown in the figure, systems containing alcohol or alkane components are highly represented in the database due to the relatively large amounts of data for these systems.

\subsection{Binary Interaction Parameter Regression}

The PR EOS binary interaction parameters for the 916 binary systems were obtained by regressing the data for each system in our database. To determine the optimum values of the BIPs in the PR EOS model, regression analyses using an equalfugacity equilibrium framework were conducted subject to mass balance constraints:

$$
\begin{aligned}
& p^{\vee}=p^{L} \\
& T^{\vee}=T^{L} \\
& \hat{f}_{i}^{\vee}=\hat{f}_{i}^{L}
\end{aligned}
$$

where $\hat{f}_{i}$ is the fugacity of component $i$ in the mixture, $T$ is the temperature, $p$ is the pressure, and the superscripts, and $\mathrm{V}$ and $\mathrm{L}$ indicate vapor and liquid phases, respectively. In the regression analyses, the EOS approach was employed for VLE systems. 


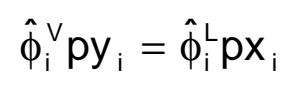

where $x_{i}$ and $y_{i}$ are the liquid and vapor mole fractions of any component $i, \hat{\phi}_{i}^{V}$ and $\hat{\phi}_{i}^{L}$ are the component fugacity coefficients in the vapor and liquid phase, respectively.

An equilibrium algorithm, GEOS [41], was used to conduct bubble-point calculations to determine the bubble-point pressures of the systems studied. A nonlinear regression procedure based on the Marquardt method $[42,43]$ was utilized in this work. Since much of the available data is in the T-p-x form (no vapor phase measurements), only the measured T-p-x data were used in the data reductions. The following objective function, OF, given below by Equation (11) was used in the regressions. $O F=\sum_{i=1}^{N D P}\left(\frac{p_{\text {cal }}-p_{\text {exp }}}{p_{\text {exp }}}\right)_{i}^{2}$

where NDP is the number of data points, $p_{\text {exp }}$ and $p_{\text {cal }}$ are the experimental and calculated bubble-point pressures, respectively.

\subsection{Descriptor Calculation}

Two-dimensional (2-D) molecular structures were generated for each component in the database using ChemDraw Ultra 11.0 [44]. Each 2-D structure was then used to generate a three-dimensional (3-D) structure. The 2-D structure can lead to several conformations of 3-D structures; however, only the 3-D conformation with the lowest energy is the stable state of the molecule. Thus, the molecular energy of the 3-D conformations was minimized by using the OpenBabel genetic algorithm-based (GA) conformal search $[45,46]$ that employs the MMFF94 force field method [47]. Details on the GA-based conformal search are summarized in the OpenBabel documentation [48]. 
The optimized molecular structures were then used to generate molecular descriptors for each component. For each component, up to 2344 molecular descriptors were generated using DRAGON [34] and 598 descriptors were generated using CODESSA [35]. Details on the descriptors calculated by DRAGON can be found elsewhere [34]. Numerous successful QSPR models based on DRAGON and CODESSA descriptors have been proposed in the literature [24-28].

\subsection{Descriptor Input Set up}

The structural descriptors generated from DRAGON [34] and CODESSA [35] were used as input values in the development of the QSPR model. For each binary system, the input descriptor set was prepared by calculating the absolute differences in the individual descriptors for the compounds in the binary pair of molecules [36]. The absolute differences were used to ensure that the QSPR model remains internally consistent; that is, if an imagined binary system consists of identical compounds, the QSPR model will correctly predict zero values for the BIPs (if $i=j, C_{i j}=D_{i j}=0$ ).

\subsection{Descriptor Reduction and Model Development}

As mentioned earlier, DRAGON and CODESSA are capable of calculating a large number of molecular descriptors for each component. However, most of these descriptors have negligible influence in describing a specific property of a component. Therefore, descriptor reduction methodologies are used to reduce the number of descriptors by finding those that are most significant for describing a given property of interest.

In the current work, a sequential regression analysis [33] was used to identify the most significant descriptors for describing the BIPs, $C_{i j}$ and $D_{i j}$. The sequential 
regression analysis identified thirty descriptors as significant in describing these parameters. The descriptors identified in this step were then used to construct an artificial neural network (ANN) model. A detailed discussion on our descriptor reduction methodology can be found in a previous work [33].

In the ANN model development process, the input dataset was divided into training, validation, internal test and external test sets that contained $50 \%, 15 \%, 10 \%$, $25 \%$ of the data, respectively. The data was divided based on the functional-group interactions to ensure that all four data sets had sufficient representation of each of the functional-group interactions present in the database. The current work uses the backpropagation algorithm for training the multi-layer perceptron network [49]. An earlystopping method $[50,51]$ was used to avoid over-fitting by the application of training and internal validation sets. During initial phases of the ANN training, the errors in both the training and validation sets decrease; however, when the ANNs begins to over-fit the data, the error for the validation set begins to increase. The ANN training was stopped after a fixed number of iterations in which the error on the cross-validation set increased continuously, and the ANN parameters at the minimum of the cross-validation error were retained. Further, to ensure optimal ANN training and avoid local minima, multiple randomizations of the data and initializations of the initial ANN parameters (weights and biases) were used [52]. The Nguyen-Widrow algorithm [53] was used to initialize weights and biases and these were updated using a Levenberg-Marquardt optimization technique [49]. A detailed description of the ANN algorithm is available in a previous work [33].

\subsection{Creating Ensembles}


An Artificial Neural Network (ANN) can sometimes be unstable and its predictive performance is dependent strongly on the training data and the training parameters. Therefore, a single outlier in the training data could have disproportional effect on the generalization ability of the final model. To prevent this, ensembling of ANNs was utilized, where the predictions of different ANNs are averaged to result in the final predictions [54-56]. In this work, the final ensemble model consisted of 20 different ANNs, each having the same descriptors as inputs but with different network architecture and weights.

\subsection{Model Validation}

The developed QSPR model was validated by employing an external test set of compounds not used in the model development process, as emphasized by Tropsha et al.[57]. The performance of the developed model on this external dataset indicates the generalization capability (a priori predictions) of the final model.

\subsection{Model Evaluation}

To evaluate the correlative and predictive capabilities of the PR EOS in modeling the systems studied, three distinct case studies were formulated. These case studies are listed in Table 1. As shown in the table, the case studies progress systematically in the number of interaction parameters used in the model. Case 1 represents an initial evaluation of the PR model in which its "raw" predictive capability (no interaction parameters) is assessed. In Case 2, the application of a single, constant interaction parameter independent of temperature, which is a common industrial practice, is considered for each binary system. Case 3 employs a separate, constant pair of interaction parameters independent of temperature for each binary system. The 
interaction parameters for each case were obtained using the objective function given by Equation (11).

As mentioned above, our VLE database consisted of 916 binary systems with a wide range of functional-group interactions, including aliphatic and aromatic hydrocarbons, water, alcohols, ethers, sulphides and nitrile compounds. Therefore, three models were studied to test the capabilities of the PR EOS and the generalized PR-QSPR model in modeling binary systems, including separately: all systems, highly non-ideal non-aqueous systems, and aqueous systems. The three models are presented in Table 2. Model 1 represents the PR EOS and PR-QSPR model evaluation for all the systems studied (916 systems). In Model 2, the aqueous systems are excluded (861 systems). Model 3 addresses the ability of the PR EOS and PR-QSPR model for the aqueous systems ( 55 systems).

Parameters in our models were determined based on the objective function, OF, of Equation (11) - the root-mean-square of the fractional deviations in pressures. In the following discussion, the results are also expressed and analyzed in terms of the rootmean-squared error (RMSE), bias and average absolute percentage deviations (\%AAD).

$$
\begin{aligned}
& \text { RMSE }=\left(\frac{\sum_{i=1}^{N D P}\left(p_{\text {cal }}-p_{\text {exp }}\right)^{2}}{N D P}\right)^{1 / 2} \\
& \text { bias }=\frac{\sum_{i=1}^{N D P}\left(p_{\text {cal }}-p_{\text {exp }}\right)}{N D P}
\end{aligned}
$$




$$
\% \mathrm{AAD}=\frac{100}{\mathrm{NDP}} \sum_{\mathrm{i}=1}^{\mathrm{NDP}}\left|\frac{\mathrm{p}_{\text {cal }}-\mathrm{p}_{\text {exp }}}{\mathrm{p}_{\exp }}\right|_{\mathrm{i}}
$$

where, NDP is the number of data points, $p_{\text {exp }}$ and $p_{\text {cal }}$ are the experimental and calculated bubble-point pressures, respectively.

\section{Results and Discussion}

Table 3 presents a summary of results for the cases listed in Table 1, where overall model statistics are given for the bubble-point pressures for the three cases. The results are classified into system-based (overall average predictions calculated based on the number of binary systems) and point-based (overall average predictions calculated based on number of data points) average.

As shown in Table 2, the prediction capability of PR EOS in the absence of interaction parameters, $C_{i j}=D_{i j}=0$ (Case 1$)$ resulted in large deviations for the predicted bubble-point pressures, especially for highly non-ideal and aqueous systems. This illustrates that interaction parameters are necessary for modeling of systems composed of dissimilar molecular species.

\subsection{Single Parameter, $C_{\mathrm{ij}}$, Representations}

The use of a single interaction parameter, $\mathrm{C}_{\mathrm{ij}}$, specific to each binary system (Case 2) provides improved results for the 916 binary systems (Model 1). As indicated

in Table 3, the results show a marked improvement compared to Case 1 with a reduction in overall errors from $18 \%$ to $5 \%$ AAD. Further, the errors decreased from $28 \%$ to $7 \%$ for highly non-ideal systems, and from $87 \%$ to $14 \%$ for aqueous systems. This demonstrates the value of interaction parameters, especially for asymmetric mixtures or mixtures containing polar components [17].

\subsection{Two Parameter $\left(C_{i j}\right.$ and $\left.D_{i j}\right)$ Representations}


To account for molecular size effects and polarity, a second interaction parameter, $D_{\mathrm{ij}}$, was introduced into the co-volume mixing rules $[58,59]$. Case 3 involves use of a separate, constant pair of values, $C_{i j}$ and $D_{i j}$, which are determined for each binary system, independent of temperature.

Table 3 presents the results obtained for PR EOS using $C_{i j}$ and $D_{i j}$ binary parameters (Case 3). While a significant improvement is observed in in comparison with Case 1, the results indicate only a minor improvement over the use of single parameter $\mathrm{C}_{\mathrm{ij}}$ (Case 2). Although the overall \%AADs for Case $3(4 \%)$ are comparable to Case 2 (5\%), a closer inspection of the results reveals that Case 3 provides some improvement in the representations for aqueous systems. Specifically, the errors for aqueous systems were about $11 \%$ for Case 3, compared to $14 \%$ for Case 2 .

Figure 3 presents the \%AAD distribution of bubble-point pressure representations for Cases 2 and 3 classified by functional-group interactions. Results for each case of each functional-group interaction are shown in variations of grey based on the error ranges given in the figure key. As shown in the figure, both cases provided comparable results when the components of a system are composed of the same functional group (diagonal elements of the triangular matrix). However, Case 2 results in higher errors for highly non-ideal systems when compared to Case 3 . This is due to the fact that the use of a single interaction parameter $\left(C_{i j}\right)$ is insufficient for systems exhibiting higher polarity or dissimilarity in the molecular species $[60,61]$. The introduction of $D_{\mathrm{ij}}$ (Case 3 ) to account for the dissimilarity in molecular size improves the results for some highly non-ideal systems. However, both Case 2 and Case 3 yielded high errors for systems containing water. Although the PR EOS sufficiently models the 
phase behavior of the pure components of these systems, the phase behavior of a mixture of these components is poorly modeled. The poor predictions for these systems may be attributed to inherent deficiencies in the applied mixing rules and, thus, mixing rules other than the classical one-fluid mixing rules may be required for such systems.

Detailed information on bubble-point pressure representations as well as values of the optimized BIPs $\left(C_{i j}\right.$ and $\left.D_{i j}\right)$ for Cases 2 and 3 for the entire database used in this study is provided in Table S1 of the Supplementary Material available with the web edition of this paper.

\subsection{PR-QSPR Generalized Predictions}

The regressed BIPs obtained from PR EOS for both Cases 2 and 3 were used as targets in the development of QSPR models. This goal was to produce model generalizations capable of predicting bubble-point pressure within two times the error from direct data regressions. As mentioned above, three models were developed and no significant difference was observed in the errors among the three models. Therefore, model 1, in which all the systems (916 systems) were included, was chosen in the current study.

In the QSPR model development process for Case 3, where both BIPs $\left(C_{i j}\right.$ and $\left.D_{i j}\right)$ have constant values, a sequential regression process was performed in order to improve the predictive capability of the generalized model. In this work, the QSPR model development started by using the initial regressed BIPs as targets ( $1^{\text {st }}$ iteration). Then, the regression process was repeated by regressing only one of the BIPs $\left(C_{i j}\right.$ or $\left.D_{i j}\right)$ while fixing the other at the generalized value from the QSPR model. The BIPS found in this step were then used as targets to develop a new QSPR model $\left(2^{\text {nd }}\right.$ 
Iteration). These alternating regression and QSPR modeling steps are repeated multiple times until no significant improvement in predictive capability is observed. The final model for this case (Case 3) was chosen after ten iterations of the regression process.

A set of 30 molecular descriptors obtained from the sequential regression model [33] was used as inputs for the ANNs in the final ensemble model. Tables 4-6 list the molecular descriptors found significant in estimating the parameters $C_{i j}($ Case 2$), C_{i j}$ and $D_{i j}$ (Case 3), respectively. The molecular descriptors were calculated using DRAGON [34] and CODESSA [35]. The descriptors listed constitute the descriptors for both the components of the system. The most significant descriptors found in predicting the BIPs were geometric, topological indices, 2D autocorrelations, quantum chemical and GETAWAY descriptors. Additional information on these descriptors may be found in the documentation of DRAGON [34].

Figures 4(a)-(b) compare the regressed $C_{i j}$ values from PR EOS with the predicted $\mathrm{C}_{\mathrm{ij}}$ values from QSPR model for Case 2 in the training and validation sets. The correlation coefficients $\left(R^{2}\right)$ between the regressed and predicted values for the training and the validation sets are 0.97 and 0.90 , respectively. The figures indicate that the predicted PR-QSPR model parameters are in good agreement with the regressed PR EOS parameters. Figure 4(d) compares the regressed $C_{i j}$ values from PR EOS with the predicted $C_{i j}$ values from QSPR model for Case 2 in the external test set. The $R^{2}$ value between the regressed and predicted values for the external test set is 0.81 . Although the level of agreement for the external set is lower than that for the training and validation sets, these results are still indicative of the capability of the developed QSPR 
model for generalized predictions on new systems not used in the model development process.

Similarly, Figures 5(a)-(c) and 6(a)-(c) compare the regressed $C_{i j}$ and $D_{i j}$ values from PR EOS with the predicted $C_{i j}$ and $D_{i j}$ values from QSPR model ( $10^{\text {th }}$ iteration model) for Case 3 in the training, validation and external test sets.

Table 7 presents the summary results for the bubble-point pressure predictions using PR-QSPR model (Case 2) for the training, validation, internal test and external test sets. The table provides the results for all systems in the database, and also for subsets composed of highly non-ideal non-aqueous systems and for aqueous systems. As expected, the generalized model predictions contain slightly higher errors than the regressed model. Nevertheless, the generalized models provide reasonable results and permit predictions for systems for which no experimental data exist. As shown in Table 7 , the errors for the bubble-point pressure predictions in all data sets were less than two times those calculated in the PR EOS regressions (Case 2). The PR-QSPR model resulted in $6.7,9.4,10.7$ and 10.5\%AADs for the training, validation, internal test and external test sets, respectively.

Figures 8(a)-(c) illustrate the generalized model predictions for equilibrium phase compositions of $\mathrm{n}$-heptane + ethylbenzene, 1,3-butadiene + butane, chloroform + methyl acetate and isopentane $+\mathrm{N}, \mathrm{N}$-dimethylformide systems, respectively. Figure 8 is based on Case 2, where $C_{i j}$ is a constant value and $D_{i j}$ equal to zero. The figures also show a comparison between the PR-QSPR generalized model and PR EOS representations with one interaction parameter. As evident from the figures, the PRQSPR generalized model developed in this work provides predictions comparable to 
direct regressions for the equilibrium phase compositions. Further, the predictions from the PR-QSPR model are in good agreement with the experimental composition values for each system. This illustrates the capability of the PR-QSPR model for predicting various types of phase behavior including highly-non ideal systems.

Table 8 shows the summary results for the bubble-point pressure predictions using PR-QSPR model (Case 3) for the training, validation, internal test and external test sets. The errors for the bubble-point pressure predictions were within about two times the errors obtained in the PR EOS regressions (Case 3), indicating that a QSPR modeling approach is effective in generalizing PR EOS binary parameters for a priori property predictions. Table 8 indicates that the bubble-point pressure predictions from Case 3 were generally similar to Case 2 . The only exception was a minor improvement in the predictions for aqueous systems, where the errors were reduced from about 22\% (Case 2) to about $19 \% \mathrm{AAD}$ (Case 3).

Although the developed QSPR models were based on bubble-point calculations, the PR EOS can be used in other types of phase equilibrium calculations such as bubblepoint temperatures and flash calculations. In order to validate the generalized model, the BIPS obtained from the QSPR model were used to predict the bubble-point temperatures and liquid-vapor compositions for the systems studied. Table 9 presents the summary results for the bubble-point pressures, bubble-point temperatures and liquid-vapor compositions from flash calculations using the PR-QSPR model. The results indicate the generalized model can provide useful predictions under different types of calculations.

Figure 7 shows the \%AAD distribution of bubble-point pressure predictions obtained 
from PR-QSPR model for Cases 2 and 3 classified by functional-group interaction. The matrix indicates that the PR-QSPR model resulted in prediction of bubble-point pressure between $5-10 \% A A D$ for most of the functional-group interactions present in the database.

Figures 9(a)-(d) illustrate the model predictions from Cases 2 and 3 for chloroform + diisopropyl ether, N,N-dimethylformide + 1-propanol , 2-methyl 1-propanol + bromobenzene, and hexane $+\mathrm{n}$-decane systems, respectively. As shown in the figure, the PR-QSPR model for Cases 2 and 3 provide comparable predictions.

\subsection{PR-QSPR Model Predictions for High-pressure Data}

The PR-QSPR model was developed based on 916 systems as mentioned earlier. An additional higher-pressure VLE database was assembled for 22 systems including nearly ideal, highly non-ideal and aqueous systems. These data were not used in the generalized model development and, thus, they provide a test of the generalization performed in this work. The generalized BIPs obtained from QSPR model were used to predict the bubble-point pressures for these systems.

Table 10 presents the high-pressure and low-pressure VLE databases for the 22 systems. The table lists the ranges of temperature and pressure for both databases. The table also presents comparison of PR-QSPR model predictions for high-pressure data and low-pressure data of these 22 systems. The results are shown for Cases 2 and 3 (Model 1). As indicated by the \%AADs in Table 9, the bubble-point pressure pressures are reasonably accurate for high-pressure data, considering that the model development was based on predominantly low-pressure data. Overall, the PR-QSPR model provided predictions with a \%AAD of 8 and 7 for Cases 2 and 3, respectively, for 
high-pressure data. In comparison, the PR-QSPR model yielded predictions with a \%AAD of about 10 for low- pressure data, as shown in Table 9.

Figures 10(a)-(d) illustrate the prediction of vapor-liquid equilibrium for hydrogen sulfide + methanol, benzene + toluene, pentane + acetone, 1-propanol $+p$-xylene systems, respectively at high pressures. The figure shows comparison between the PRQSPR model predictions from Cases 2 and 3. As shown in Figure 10(a) for benzene + toluene system, the PR-QSPR models for both Cases 2 and 3 provide comparable predictions since the components of the system have same functional groups and the system is nearly-ideal. However, when the components of a system belong to different functional groups, Case 3, which accounts for the dissimilarity in molecular sizes, provides better predictions than Case 2 as shown in Figure 10(b) for hydrogen sulfide + methanol system. For 1-propanol + p-xylene system shown in Figure 10(d), both cases provide good predictions at $334 \mathrm{~K}$ compared to the experimental data. However, the generalized predictions for both cases show larger errors at $494 \mathrm{~K}$, especially when the mole fraction of 1-propanol is more than $80 \%$; however, this high temperature is near the critical temperature of the system.

\subsection{BIPs Prediction for PR EOS from Different Methods}

Several approaches, empirical as well as semi-empirical, have been presented in the literature to estimate the binary interaction parameters. These include simpler correlations using structural parameters. Although these correlations are easier to implement, they are limited by their range of applicability and/or by poor suitability for

generalization. In earlier works, we developed several correlations for the BIPs of PR EOS and the correlations were capable of describing the phase behavior of several 
systems [16-19]. In this work, our aim is to advance the method by developing reliable, generalized models applicable for much more diverse range of mixtures.

A comparison of the PR EOS predictions using different generalization methods presented in the literature indicates that applicability of the previous methods is limited. In particular, Gasem [17] developed generalized correlations for the binary interaction parameters, Cij and $\mathrm{Dij}$, in terms of paraffin carbon number, the acentric factor and temperature. This work was limited to $\mathrm{CO}_{2}$ and $\mathrm{n}$-paraffin systems. Further, the quality of the predictions obtained using such correlations is dependent on the complexity of the generalization scheme used. Gasem et al. [18] developed similar correlations for the BIPs to represent the phase behavior of ethane $+n$-paraffin and $\mathrm{CO}_{2}+n$-paraffin systems. These correlations provide prediction of the bubble point pressures with an error of about $5.7 \%$ (0.014 in mole fraction). Nishiumi et al. [62] developed a correlation for $\mathrm{C}_{\mathrm{ij}}$ in terms of critical volume and acentric factor. The correlation was applied to calculate the binary interaction parameters of more than 100 binary systems. However, their work was limited to hydrocarbons, $\mathrm{N}_{2}, \mathrm{CO}_{2}$ and hydrogen sulfide systems. The phase equilibria of similar systems were also investigated by Fateen et al. [63]. They presented the interaction parameter, $\mathrm{C}_{\mathrm{ij}}$, for PR EOS as function of temperatures and pressures. In our previous work [16], we developed generalized correlations in terms of temperature for predicting the high-pressure phase equilibria of systems of coalbed gases (methane, nitrogen and carbon dioxide) with water. The predictions using the temperature-dependent expressions yielded average absolute percentage errors of about $3-6 \%$ for these systems. In fact, numerous correlations exist in the literature and an attempt to list all the available correlations is not made here. Since most of these 
correlations were limited to specific systems, a direct comparison between these correlations and our QSPR model with much wider applicability in terms of mixtures represented would not be very meaningful.

In summary, the generalized PR-QSPR model presented in this study has been shown to provide predictions for the bubble-point pressures within twice the errors calculated in the PR EOS regression analysis for both cases 2 and 3 . This was demonstrated by testing the generalized PR-QSPR model for a variety of binary systems composed of diverse molecular species over range of temperatures and pressures. As evident from the above results, cases 2 and 3 provide comparable predictions. However, Case 3 was generally superior to Case 2 for systems exhibiting higher polarity or dissimilarity in the molecular species.

\section{Conclusions}

The PR EOS with the classical one-fluid mixing rules was employed to model the vapor-liquid equilibria of 916 binary systems composed of diverse molecular species. QSPR modeling approach was applied to generalize the binary interaction parameter of PR EOS model. A total of 30 molecular structural descriptors were utilized as inputs in the QSPR model.

Results indicate that the PR-QSPR model is capable of providing useful predictions of bubble-point pressures of binary systems. In particular, the results reveal that the predicted bubble-point pressures from the PR-QSPR model were within twice the errors calculated in the PR EOS regression analysis. The generalized model was further validated by predicting bubble-point pressures for high-pressure VLE data of 22 systems. The model provided predictions for these data comparable to predictions 
obtained for the low-pressure data used in model development. Thus, the PR-QSPR model developed in this work has been shown capable for a priori predictions of VLE for

a diverse set of binary systems over wide conditions of pressure, temperature and composition.

Acknowledgment

We gratefully acknowledge the financial support received from the U.S. Department of Energy and the Coal-Seq Consortium. 


\section{References}

[1] S. Zeck, D. Wolf, Requirements of thermodynamic data in the chemical industry, Fluid Phase Equilibria, 82 (1993) 27-38.

[2] P.M. Mathias, T.W. Copeman, Extension of the Peng-Robinson equation of state to complex mixtures: Evaluation of the various forms of the local composition concept, Fluid Phase Equilibria, 13 (1983) 91-108.

[3] R.A. Heidemann, A.M. Khalil, The calculation of critical points, AIChE Journal, 26 (1980) 769-779.

[4] D. Abrams, F. Seneci, P. Chueh, J. Prausnitz, Thermodynamics of multicomponent liquid mixtures containing subcritical and supercritical components, Industrial \& Engineering Chemistry Fundamentals, 14 (1975) 52-54.

[5] G. Soave, Equilibrium constants from a modified Redlich-Kwong equation of state, Chemical Engineering Science, 27 (1972) 1197-1203.

[6] D.Y. Peng, D.B. Robinson, A new two-constant equation of state, Industrial \& Engineering Chemistry Fundamentals, 15 (1976) 59-64.

[7] G.F. Chou, J.M. Prausnitz, A phenomenological correction to an equation of state for the critical region, AIChE Journal, 35 (1989) 1487-1496.

[8] P.M. Mathias, H.C. Klotz, J.M. Prausnitz, Equation-of-state mixing rules for multicomponent mixtures: the problem of invariance, Fluid Phase Equilibria, 67 (1991) $31-44$.

[9] J. Schwartzentruber, H. Renon, Equations of state: how to reconcile flexible mixing rules, the virial coefficient constraint and the "Michelsen-Kistenmacher syndrome" for multicomponent systems, Fluid Phase Equilibria, 67 (1991) 99-110.

[10] S.K. Shibata, S.I. Sandler, Critical evaluation of equation of state mixing rules for the prediction of high-pressure phase equilibria, Industrial and Engineering Chemistry Research, 28 (1989) 1893-1898. 
[11] P.T. Eubank, G.-S. Shyu, N.S.M. Hanif, New procedures for application of the Wong-Sandler mixing rules to the prediction of vapor-liquid equilibria, Industrial and Engineering Chemistry Research, 34 (1995) 314-323.

[12] H. Orbey, S.I. Sandler, Reformulation of Wong-Sandler mixing rule for cubic equations of state, AIChE Journal, 41 (1995) 683-690.

[13] C.H. Twu, J.E. Coon, J.R. Cunningham, A new generalized alpha function for a cubic equation of state Part 1. Peng-Robinson equation, Fluid Phase Equilibria, 105 (1995) 49-59.

[14] D.S.H. Wong, S.I. Sandler, A theoretically correct mixing rule for cubic equations of state, AIChE Journal, 38 (1992) 671-680.

[15] N. Trivedi, M. S. Thesis, in, Oklahoma State University, Stillwater, OK, 1996.

[16] A.M. Abudour, S.A. Mohammad, K.A.M. Gasem, Modeling high-pressure phase equilibria of coalbed gases/water mixtures with the Peng-Robinson equation of state, Fluid Phase Equilibria, 319 (2012) 77-89.

[17] K.A.M. Gasem, Binary vapor-liquid phase equilibrium for carbon dioxide + heavy normal paraffins (interaction parameters, density predictions, pure hydrocarbon properties), in, Oklahoma State University, United States -- Oklahoma, 1986.

[18] K.A.M. Gasem, C.H. Ross, R.L. Robinson, Prediction of ethane and $\mathrm{CO}_{2}$ solubilities in heavy norma paraffins using generalized-parameter Soave and Peng-Robinson equations of state, The Canadian Journal of Chemical Engineering, 71 (1993) 805-816.

[19] W. Gao, R.L. Robinson Jr, K.A.M. Gasem, Alternate equation of state combining rules and interaction parameter generalizations for asymmetric mixtures, Fluid Phase Equilibria, 213 (2003) 19-37.

[20] J.-N. Jaubert, F. Mutelet, VLE predictions with the Peng-Robinson equation of state and temperature dependent kij calculated through a group contribution method, Fluid Phase Equilibria, 224 (2004) 285-304.

[21] J.-N. Jaubert, S. Vitu, F. Mutelet, J.-P. Corriou, Extension of the PPR78 model (predictive 1978, Peng-Robinson EOS with temperature dependent kij calculated 
through a group contribution method) to systems containing aromatic compounds, Fluid Phase Equilibria, 237 (2005) 193-211.

[22] S. Vitu, J.-N. Jaubert, F. Mutelet, Extension of the PPR78 model (Predictive 1978, Peng-Robinson EOS with temperature dependent kij calculated through a group contribution method) to systems containing naphtenic compounds, Fluid Phase Equilibria, 243 (2006) 9-28.

[23] M. Lashkarbolooki, Z.S. Shafipour, A.Z. Hezave, H. Farmani, Use of artificial neural networks for prediction of phase equilibria in the binary system containing carbon dioxide, The Journal of Supercritical Fluids, 75 (2013) 144-151.

[24] A.R. Katritzky, L. Mu, M. Karelson, QSPR treatment of the unified nonspecific solvent polarity scale, Journal of Chemical Information and Computer Sciences, 37 (1997) 756-761.

[25] A.R. Katritzky, V.S. Lobanov, M. Karelson, Normal boiling points for organic compounds: $\square$ correlation and prediction by a quantitative structure-property relationship, Journal of Chemical Information and Computer Sciences, 38 (1998) 28-41.

[26] M. Goodarzi, T. Chen, M.P. Freitas, QSPR predictions of heat of fusion of organic compounds using Bayesian regularized artificial neural networks, Chemometrics and Intelligent Laboratory Systems, 104 (2010) 260-264.

[27] M. Bagheri, K. Yerramsetty, K.A.M. Gasem, B.J. Neely, Molecular modeling of the standard state heat of formation, Energy Conversion and Management, 65 (2013) 587596.

[28] F. Gharagheizi, QSPR Studies for Solubility Parameter by Means of Genetic Algorithm-Based Multivariate Linear Regression and Generalized Regression Neural Network, QSAR \& Combinatorial Science, 27 (2008) 165-170.

[29] M. Shacham, G.S. Cholakov, R.P. Stateva, N. Brauner, Quantitative Structure-Property Relationships for Prediction of Phase Equilibrium Related Properties, Industrial \& Engineering Chemistry Research, 49 (2009) 900-912.

[30] K.A.M. Gasem, W. Gao, Z. Pan, R.L. Robinson, Jr., A Modified Temperature Dependence for the Peng-Robinson Equation of State, Fluid Phase Equilibria, 181 (2001) 113-125. 
[31] J.M. Prausnitz, R.N. Lichtenthaler, E.G. Azevedo, Molecular Thermodynamics of Fluid-Phase Equilibria, 3rd ed., Prentice-Hall, New Jersey, 1999.

[32] C. Tsonopoulos, J.L. Heidman, High-pressure vapor-liquid equilibria with cubic equations of state, Fluid Phase Equilibria, 29 (1986) 391-414.

[33] S. Golla, S. Madihally, R.L. Robinson Jr, K.A.M. Gasem, Quantitative structureproperty relationship modeling of skin sensitization: A quantitative prediction, Toxicology in Vitro, 23 (2009) 454-465.

[34] T. SRL, Dragon Professional 6.0.9, in, 2011.

[35] A.R. Katritzky, V.L. Lobanov, M. Karelson, Codessa 2.7.8, in, 2007.

[36] S. Gebreyohannes, Y. Dadmohammadi, B.J. Neely, K.A.M. Gasem, A comparative study of QSPR generalized activity coefficient model parameters for VLE mixtures, Under review (2014).

[37] U.O. J. Gmehling, W. Arlt,, Vapor-liquid equilibrium data collection, chemistry data series, in: DECHEMA (Ed.), Frankfurt, Germany, 1977 - 2001.

[38] NIST standard reference database 103b thermodata engine, in: NIST-TDE (Ed.), 2012.

[39] Physical and thermodynamic properties of pure chemicals, in: DIPPR Project 801, 2011.

[40] J. Gmehling, J. Li, M. Schiller, A modified UNIFAC model. 2. Present parameter matrix and results for different thermodynamic properties, Industrial \& Engineering Chemistry Research, 32 (1993) 178-193.

[41] K.A.M. Gasem, GEOS, in, Oklahoma State university, Stillwater, OK, 1988-1999.

[42] D.W. Marquardt, An algorithm for least-squares estimation of nonlinear parameters, Journal of the Society for Industrial and Applied Mathematics, 11 (1963) 431-441. 
[43] L.W. Jackson, A comparison of selected gradient methods for solving the nonlinear least squares problem, in: Computing and Information Sciences, Oklahoma State University, Stillwater, OK, 1978.

[44] CambridgeSoft, ChemBiooffice 11.0, in, 2008.

[45] R. Guha, M.T. Howard, G.R. Hutchison, P. Murray-Rust, H. Rzepa, C. Steinbeck, J. Wegner, E.L. Willighagen, The blue obeliskinteroperability in chemical informatics, Journal of Chemical Information and Modeling, 46 (2006) 991-998.

[46] Open Babel Package, in, http://openbabel.sourceforge.net/. 2011.

[47] T.A. Halgren, Merck molecular force field. I. Basis, form, scope, parameterization, and performance of MMFF94, Journal of Computational Chemistry, 17 (1996) 490-519.

[48] The Open Babel Developers, in: OBConformerSearch Class Reference, http://openbabel.org/dev-api/classOpenBabel11OBConformerSearch.shtml, 2007 (cited 26.07.11).

[49] H. Demuth, M. Beale, M. Hagan, Neural Network Toolbox, in, MathWorks, Inc., 2010.

[50] L. Prechelt, Automatic early stopping using cross validation: quantifying the criteria, Neural Networks, 11 (1998) 761-767.

[51] C. Rich, L. Steve, G. Lee, Overfitting in neural nets: backpropagation, conjugate gradient, and early stopping, in, 2000.

[52] M.S. Iyer, R.R. Rhinehart, A method to determine the required number of neuralnetwork training repetitions, Neural Networks, IEEE Transactions on, 10 (1999) 427432.

[53] D. Nguyen, B. Widrow, Improving the learning speed of 2-layer neural networks by choosing initial values of the adaptive weights, in: Proceedings of the International Joint Conference on Neural Networks, 1990, pp. 21-26.

[54] K.M. Yerramsetty, Quantitative structure-property relationship modeling \& computer-aided molecular designimprovements \& applications /by Krishna M. 
Yerramsetty, in: Chemical Engineering, Oklahoma State University, Stillwater, 2012, pp. 290.

[55] D.K. Agrafiotis, W. Cedeño, V.S. Lobanov, On the use of neural network ensembles in QSAR and QSPR, Journal of Chemical Information and Computer Sciences, 42 (2002) 903-911.

[56] C. Merkwirth, H. Mauser, T. Schulz-Gasch, O. Roche, M. Stahl, T. Lengauer, Ensemble methods for classification in cheminformatics, Journal of Chemical Information and Computer Sciences, 44 (2004) 1971-1978.

[57] A. Tropsha, P. Gramatica, V.K. Gombar, The importance of being earnest: Validation is the absolute essential for successful application and interpretation of QSPR models, QSAR Comb. Sci., 22 (2003) 69-77.

[58] E.A. Turek, R. S. Metcalfe, L. Yarborough and R. L. Robinson, Phase equilibria in CO2-multicomponent hydrocarbon systems: experimental data and an improved prediction technique, Society of Petroleum Engineers journal, 24 (1984) 308-324.

[59] K.A.M. Gasem, R.L. Robinson Jr., Prediction of phase behavior for $\mathrm{CO}_{2}$ plus heavy normal paraffins using generalized-parameter Soave and Peng-Robinson equations of state, in: Proceedings of the 1985 AICHE Spring National Meeting and Petro Expo '85, Houston, TX, 1985.

[60] P.M. Mathias, A versatile phase equilibrium equation of state, Industrial \& Engineering Chemistry Process Design and Development, 22 (1983) 385-391.

[61] J.M. Prausnitz, Phase equilibria and fluid properties in the chemical industry, AIChE Proceedings 2nd International Cong, Part 2, (1980) 231.

[62] H. Nishiumi, T. Arai, K. Takeuchi, Generalization of the binary interaction parameter of the Peng-Robinson equation of state by component family, Fluid Phase Equilibria, 42 (1988) 43-62.

[63] S.-E.K. Fateen, M.M. Khalil, A.O. Elnabawy, Semi-empirical correlation for binary interaction parameters of the Peng-Robinson equation of state with the van der Waals mixing rules for the prediction of high-pressure vapor-liquid equilibrium, Journal of Advanced Research, 4 (2013) 137-145. 
[64] C.-B. Soo, E.E. Ahmar, C. Coquelet, D. Ramjugernath, D. Richon, Vapor-liquid equilibrium measurements and modeling of the n-butane + ethanol system from 323 to 423 K, Fluid Phase Equilibria, 286 (2009) 79-87.

[65] K. Hynynen, P. Uusi-Kyyny, J.-P. Pokki, M. Pakkanen, J. Aittamaa, Isothermal vapor liquid equilibrium for 2-methylpropene + methanol, + 1-propanol, + 2-propanol, + 2-butanol, and + 2-methyl-2-propanol binary systems at $364.5 \mathrm{~K}$, Journal of Chemical \& Engineering Data, 51 (2006) 562-568.

[66] W.B. Kay, R.L. Hoffman, O. Davies, Vapor-liquid equilibrium relations of binary systems n-butane-n-pentane and n-butane-n-hexane, Journal of Chemical \& Engineering Data, 20 (1975) 333-338.

[67] S.W. Campbell, R.A. Wilsak, G. Thodos, (Vapor + liquid) equilibrium behavior of (npentane + ethanol) at 372.7, 397.7, and $422.6 \mathrm{~K}$, The Journal of Chemical Thermodynamics, 19 (1987) 449-460.

[68] A.-D. Leu, J.J. Carroll, D.B. Robinson, The equilibrium phase properties of the methanol-hydrogen sulfide binary system, Fluid Phase Equilibria, 72 (1992) 163-172.

[69] J. Griswold, D. Andres, V. Klein, Determination of high-pressure vapor-liquid equilibria. The vapor-liquid equilibrium of benzene-toluene, Trans. Am. Inst. Chem. Eng, 39 (1943) 223.

[70] Y. Kim, W. Bae, H. Kim, Isothermal vapor-liquid equilibria for the n-pentane + 1butanol and n-pentane +2 -butanol systems near the critical region of the mixtures, Journal of Chemical \& Engineering Data, 50 (2005) 1520-1524.

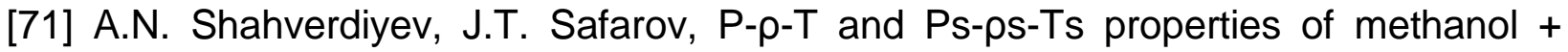
water and $n$-propanol + water solutions in wide range of state parameters, Physical Chemistry Chemical Physics, 4 (2002) 979-986.

[72] P. Sentenac, Y. Bur, E. Rauzy, C. Berro, Density of Methanol + Water between 250 $\mathrm{K}$ and $440 \mathrm{~K}$ and up to $40 \mathrm{MPa}$ and Vapor-Liquid Equilibria from $363 \mathrm{~K}$ to $440 \mathrm{~K}$, Journal of Chemical \& Engineering Data, 43 (1998) 592-600.

[73] F. Barr-David, B.F. Dodge, Vapor-liquid equilibrium at high pressures. The systems ethanol-water and 2-propanol-water, Journal of Chemical \& Engineering Data, 4 (1959) 107-121. 
[74] D.S.K. Tsiklis, A. I.; Shenderei, L. I. , Phase equilibria in the system ethanol + ethylene + water at high pressures and temperatures, Khim. Prom-st. (Moscow), No. 5 (1960) 401-406.

[75] S.W. Campbell, R.A. Wilsak, G. Thodos, Isothermal vapor-liquid equilibrium measurements for the n-pentane-acetone system at 372.7, 397.7, and $422.6 \mathrm{~K}$, Journal of Chemical \& Engineering Data, 31 (1986) 424-430.

[76] J.M. Lenoir, H.G. Hipkin, Measured enthalpies for mixtures of benzene with npentane, Journal of Chemical \& Engineering Data, 17 (1972) 319-323.

[77] V.N.K. Rao, D.R. Swami, M.N. Rao, Vapor-liquid equilibria of benzene-n-hexane and benzene-cyclohexane systems, AIChE Journal, 3 (1957) 191-197.

[78] M.S. Medani, M.A. Hasan, Thermodynamic properties of the $n$-hexane and benzene system at elevated temperatures, Journal of Applied Chemistry and Biotechnology, 27 (1977) 80-92.

[79] K.L. Butcher, K.R. Ramasubramanian, M.S. Medani, Thermodynamic properties of the benzene and $n$-heptane system at elevated temperatures, Journal of Applied Chemistry and Biotechnology, 22 (1972) 1139-1155.

[80] A. Deák, A.I. Victorov, T.W. de Loos, High pressure VLE in alkanol + alkane mixtures. Experimental results for n-butane + ethanol, +1-propanol, +1-butanol systems and calculations with three EOS methods, Fluid Phase Equilibria, 107 (1995) 277-301.

[81] A. Zawisza, J. Vejrosta, High-pressure liquid-vapour equilibria, critical state, and $\mathrm{p}(\mathrm{V}, \mathrm{T}, \mathrm{x})$ up to $573.15 \mathrm{~K}$ and $5.066 \mathrm{MPa}$ for (heptane+propan-1-ol), The Journal of Chemical Thermodynamics, 14 (1982) 239-249.

[82] J. Oison, Thermodynamics of hydrogen-bonding mixtures 2. G E, H E, andS E of 1propanol+ n-heptane, International journal of thermophysics, 16 (1995) 215-226.

[83] A.-Q. Chen, G.J. Urbanus, K.-C. Chao, A new vapor-liquid equilibrium cell and VLE data for mixtures of 1-propanol+p-xylene, Fluid Phase Equilibria, 94 (1994) 281-288.

[84] K.L. Butcher, M.S. Medani, Thermodynamic properties of methanol-benzene mixtures at elevated temperatures, Journal of Applied Chemistry, 18 (1968) 100-107. 
[85] J.R. Khurma, O. Muthu, S. Munjal, B.D. Smith, Total-pressure vapor-liquid equilibrium data for binary systems of dichloromethane with pentane, acetone, ethyl acetate, methanol, and acetonitrile, Journal of Chemical \& Engineering Data, 28 (1983) 412-419.

[86] M.S.K. Rozhnov, Phase and volume relationships in the butadiene plus hydrocarbon systems Prom-st. (Moscow), 43 (1967) 48.

[87] A.D. Leu, D.B. Robinson, Equilibrium phase properties of the n-butane-hydrogen sulfide and isobutane-hydrogen sulfide binary systems, Journal of Chemical \& Engineering Data, 34 (1989) 315-319. 
Table 1. Specific Cases Used in the Peng-Robinson

\section{Equation of State Evaluations}

\begin{tabular}{|l|l|}
\hline Case & Description \\
\hline 1. $C_{i j}=0, D_{i j}=0$ & $\begin{array}{l}\text { This provides the "raw" predictive capability of the } \\
\text { model, using the mixing rules with no interaction } \\
\text { parameters. }\end{array}$ \\
\hline 2. $C_{i j}=$ Constant, $D_{i j}=0$ & $\begin{array}{l}\text { A single, constant value } C_{i j} \text { is determined for each } \\
\text { binary system, independent of temperature. }\end{array}$ \\
\hline 3. $C_{i j}=$ Constant, $D_{i j}=$ Constant & $\begin{array}{l}\text { A separate, constant pair of values, } C_{i j} \text { and } D_{i j}, \text { is } \\
\text { determined for each binary system, independent } \\
\text { of temperature. }\end{array}$ \\
\hline
\end{tabular}

\section{Table 2. Models Used in the Peng-Robinson Equation of State and PR-QSPR Model Evaluations}

\begin{tabular}{|c|c|l|}
\hline Model & $\begin{array}{c}\text { No. of } \\
\text { systems }\end{array}$ & Description \\
\hline 1. & 916 & All 916 binary systems were included in the modeling process \\
\hline 2. & 861 & Aqueous systems were excluded in the modeling process \\
\hline 3. & 55 & Only aqueous systems were included in the modeling process \\
\hline
\end{tabular}




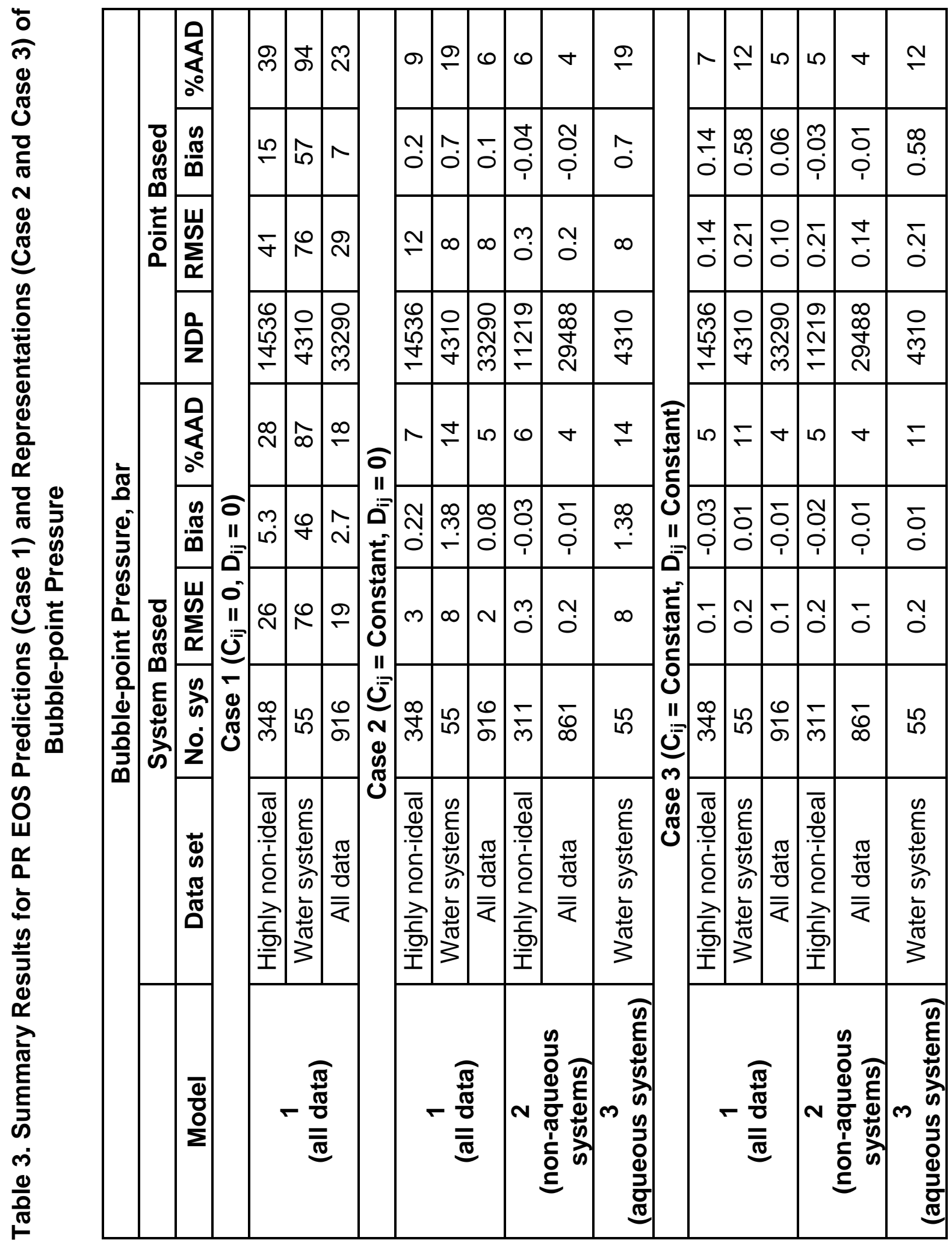



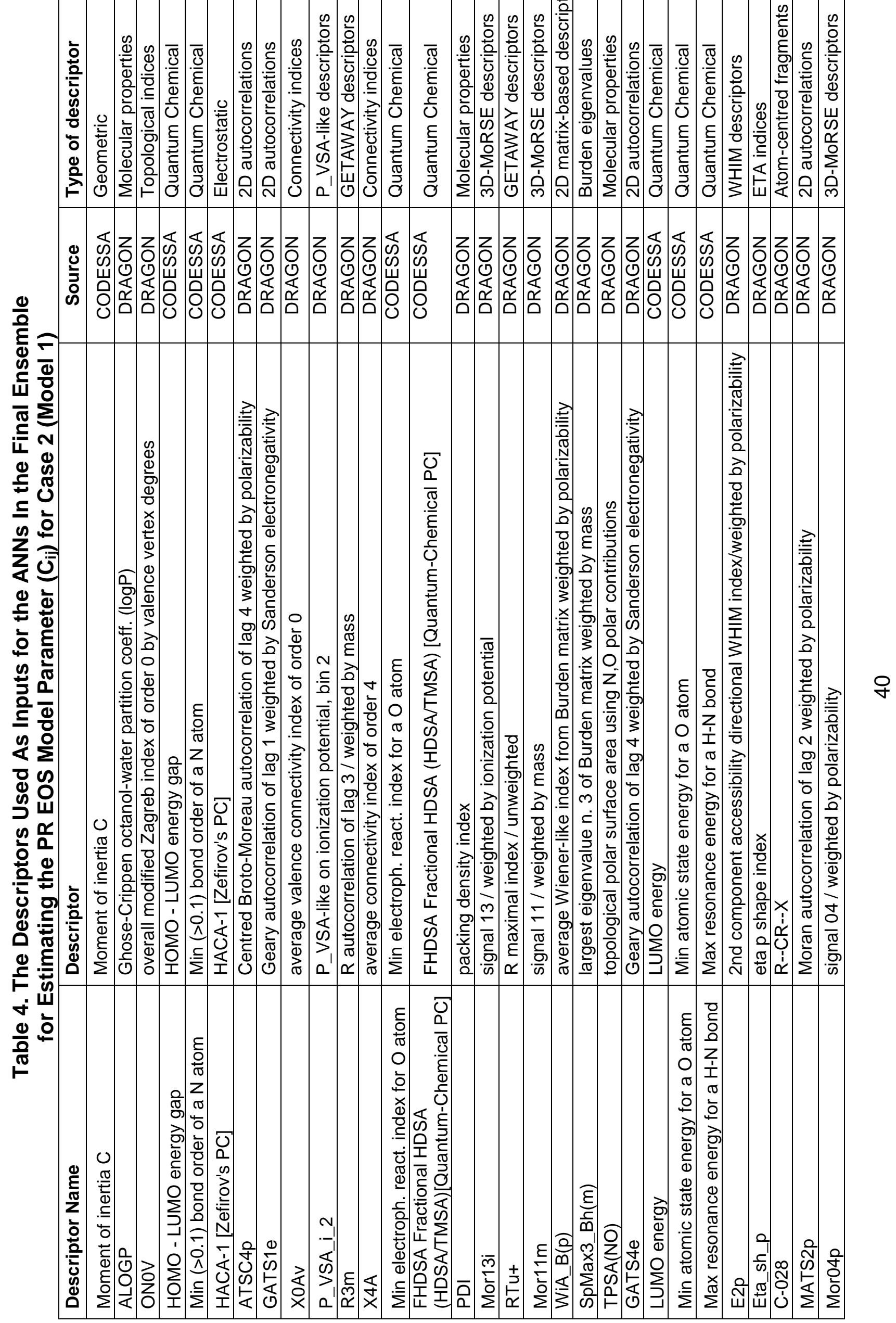


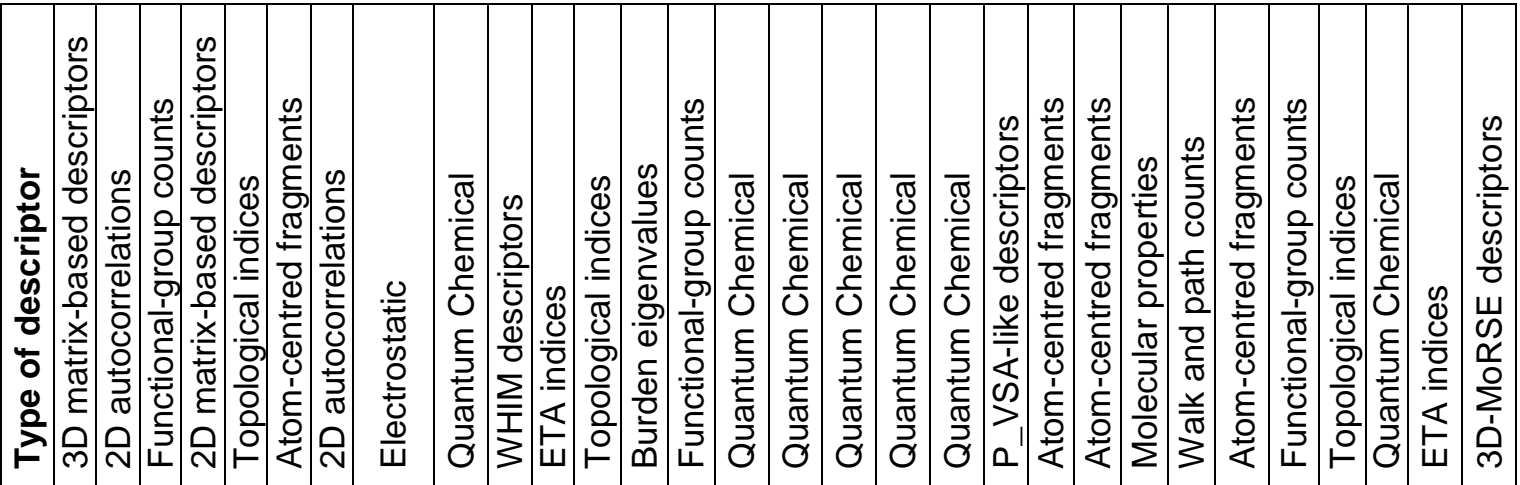

zzzzzzz⿺⿻一⿰冫⿰亅⿱丿丶丶⿱⿰㇒一乂

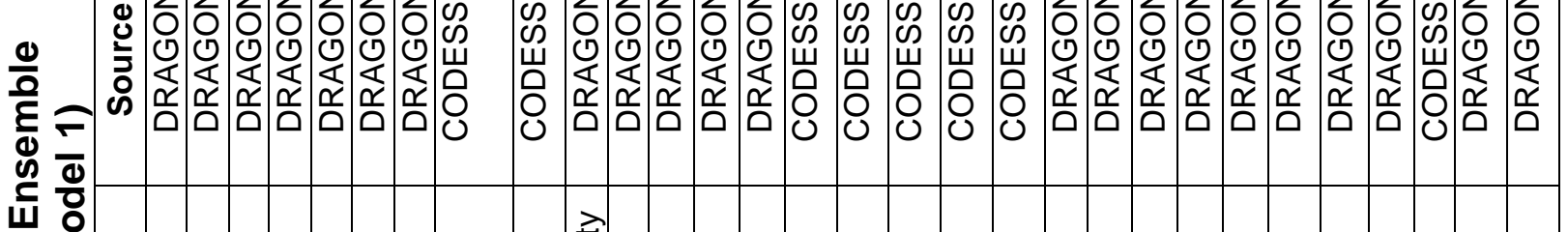

$\frac{\pi}{2}$

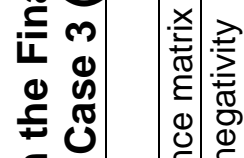

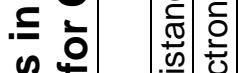

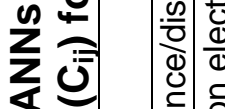

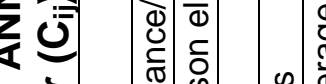

$\stackrel{0}{\rightleftharpoons}$

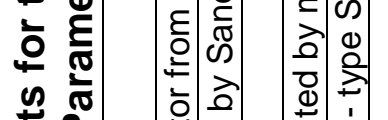

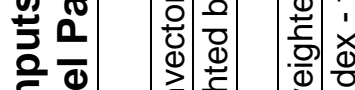

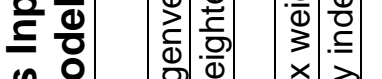

क $\frac{0}{2}$ 의

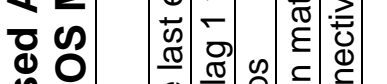

乌ั ய

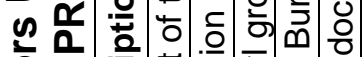

은 을

은 至

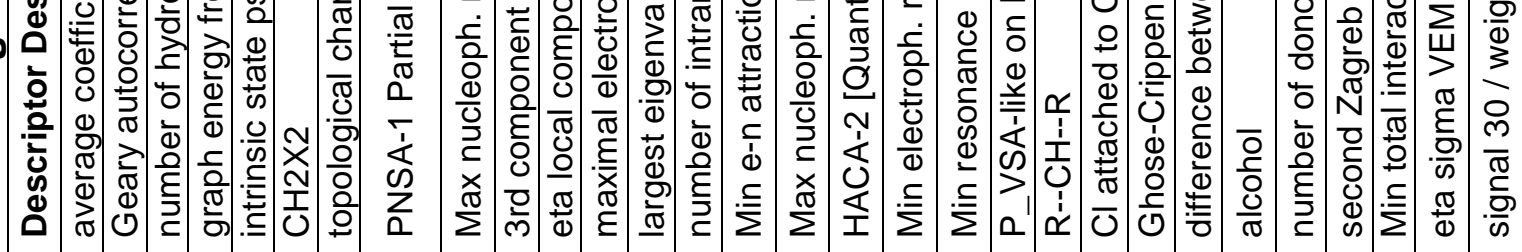

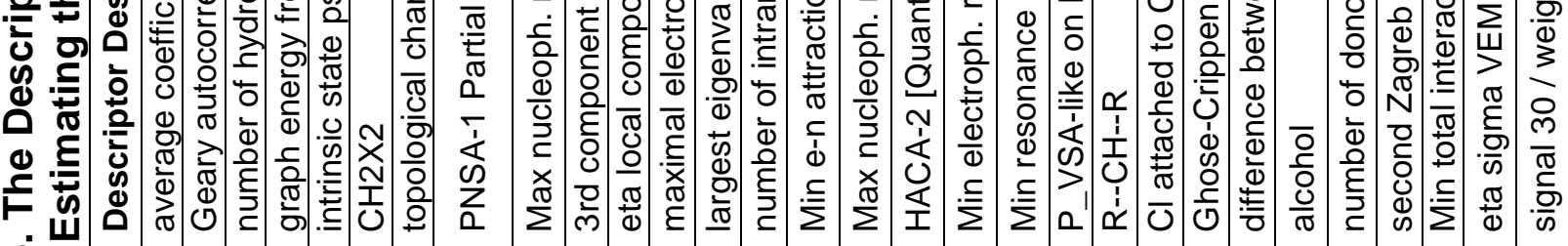

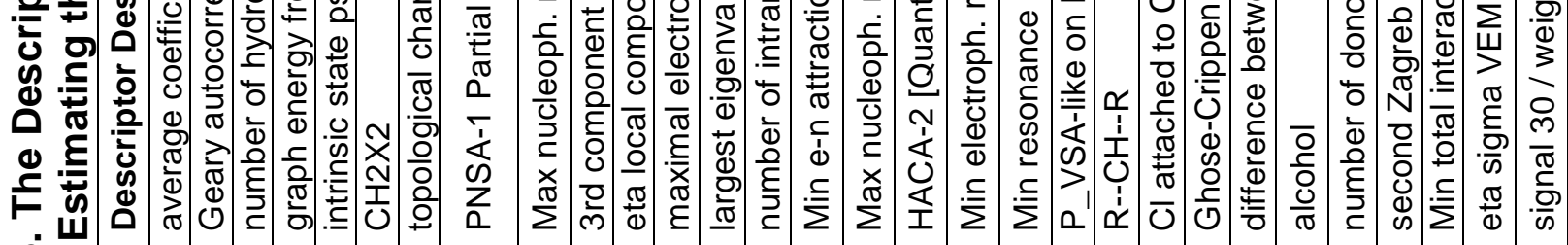

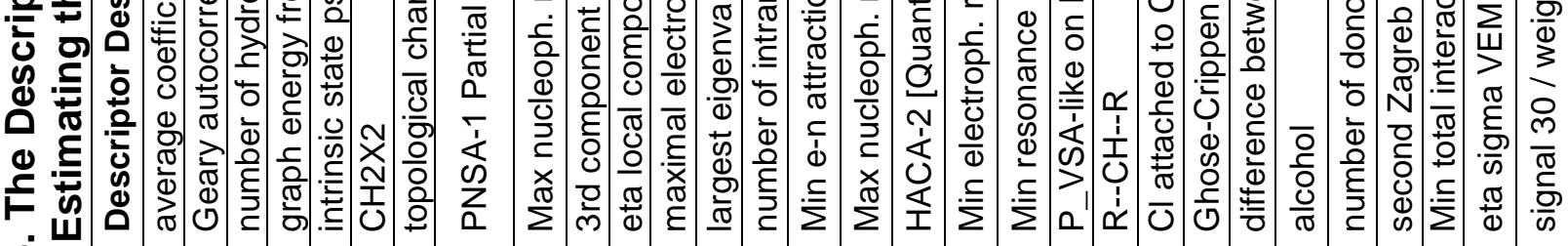
$\frac{0}{\frac{0}{0}}$

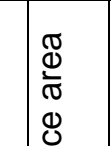

竞

흐 $\quad$ ही

은 仓)

至

๖

突
흘

근

음

ลิ

옹

$\frac{3}{3}$

× $\quad$ D

.든

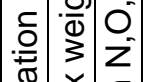

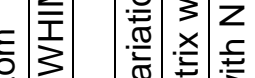

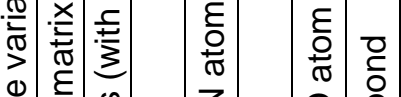

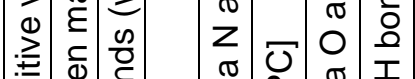

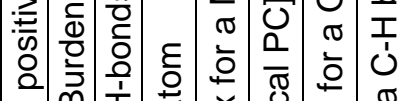

×

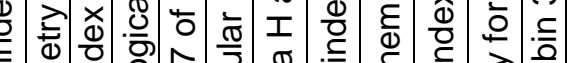

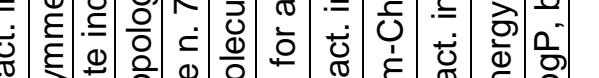

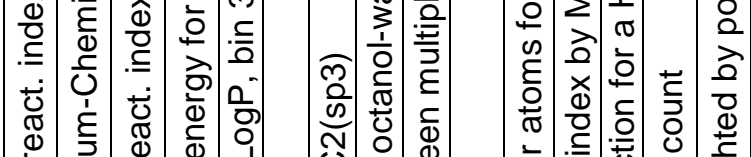

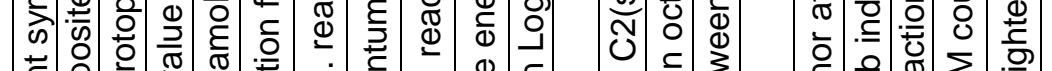

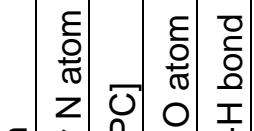

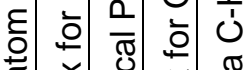

I

๙

ఫे

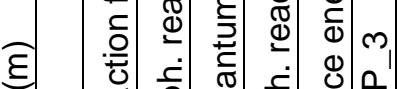

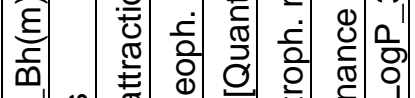

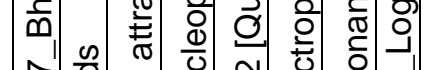

응

Ò

힌

드

은

Фั

$\stackrel{ \pm}{.} \infty$ 

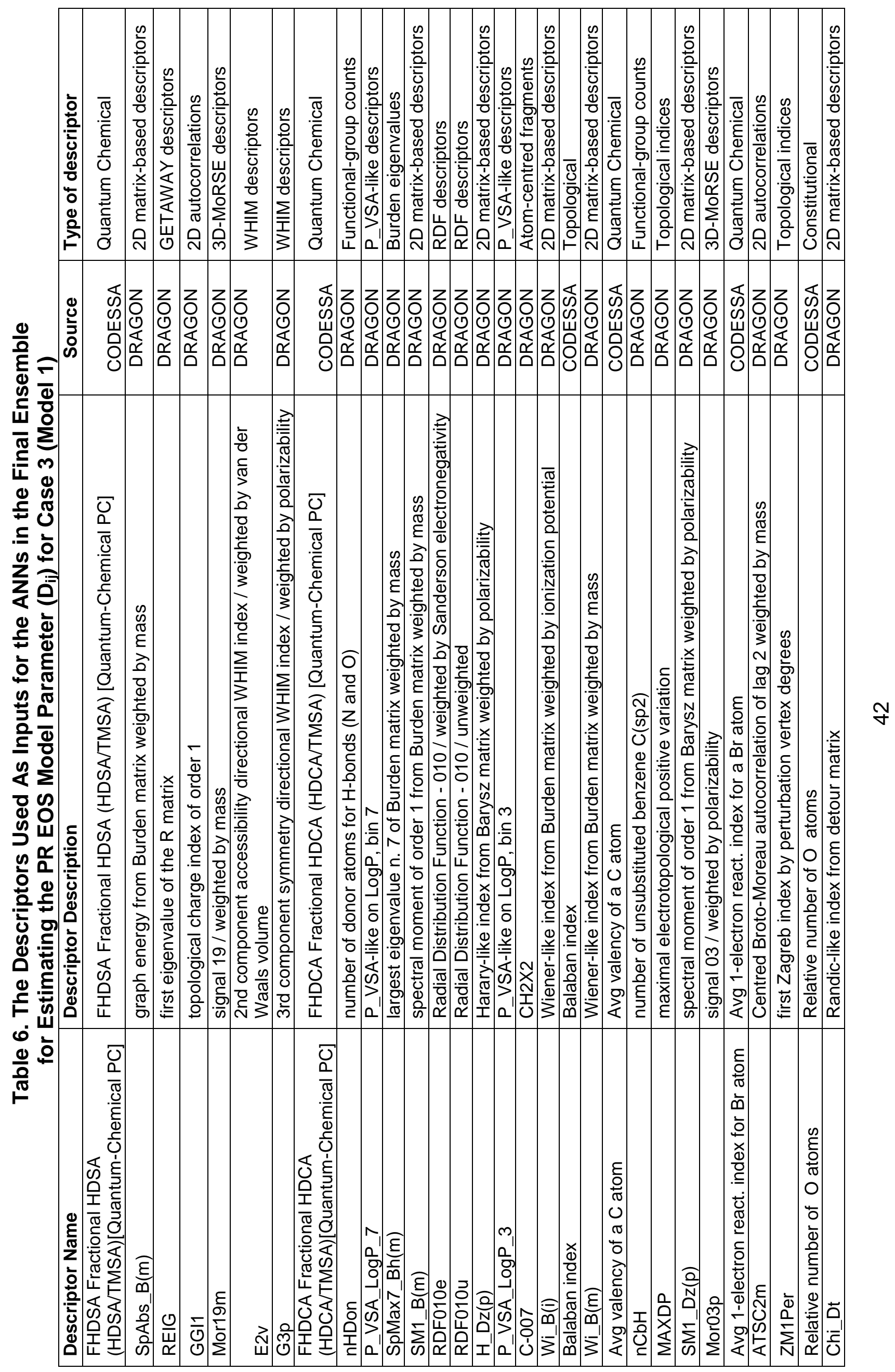


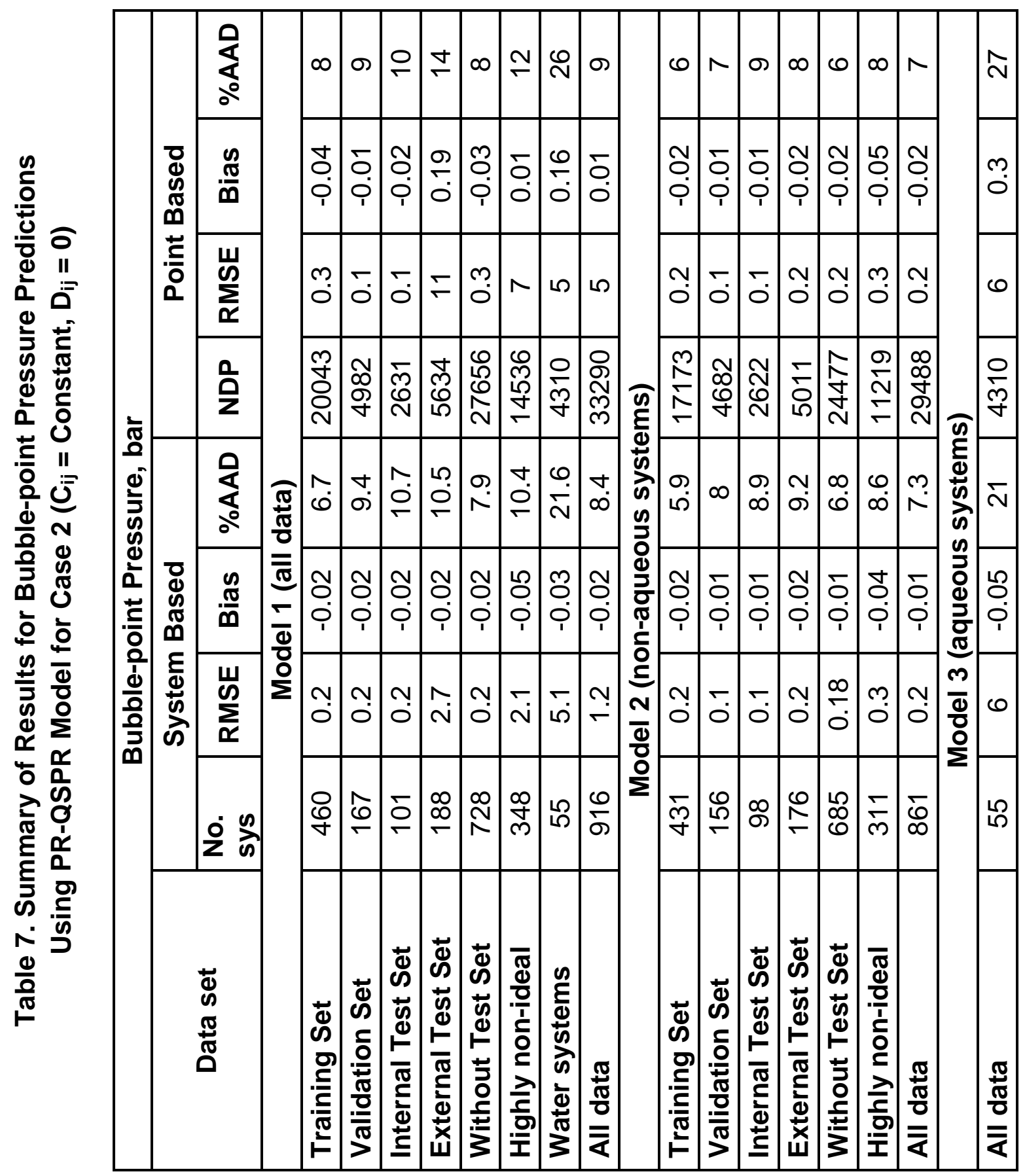




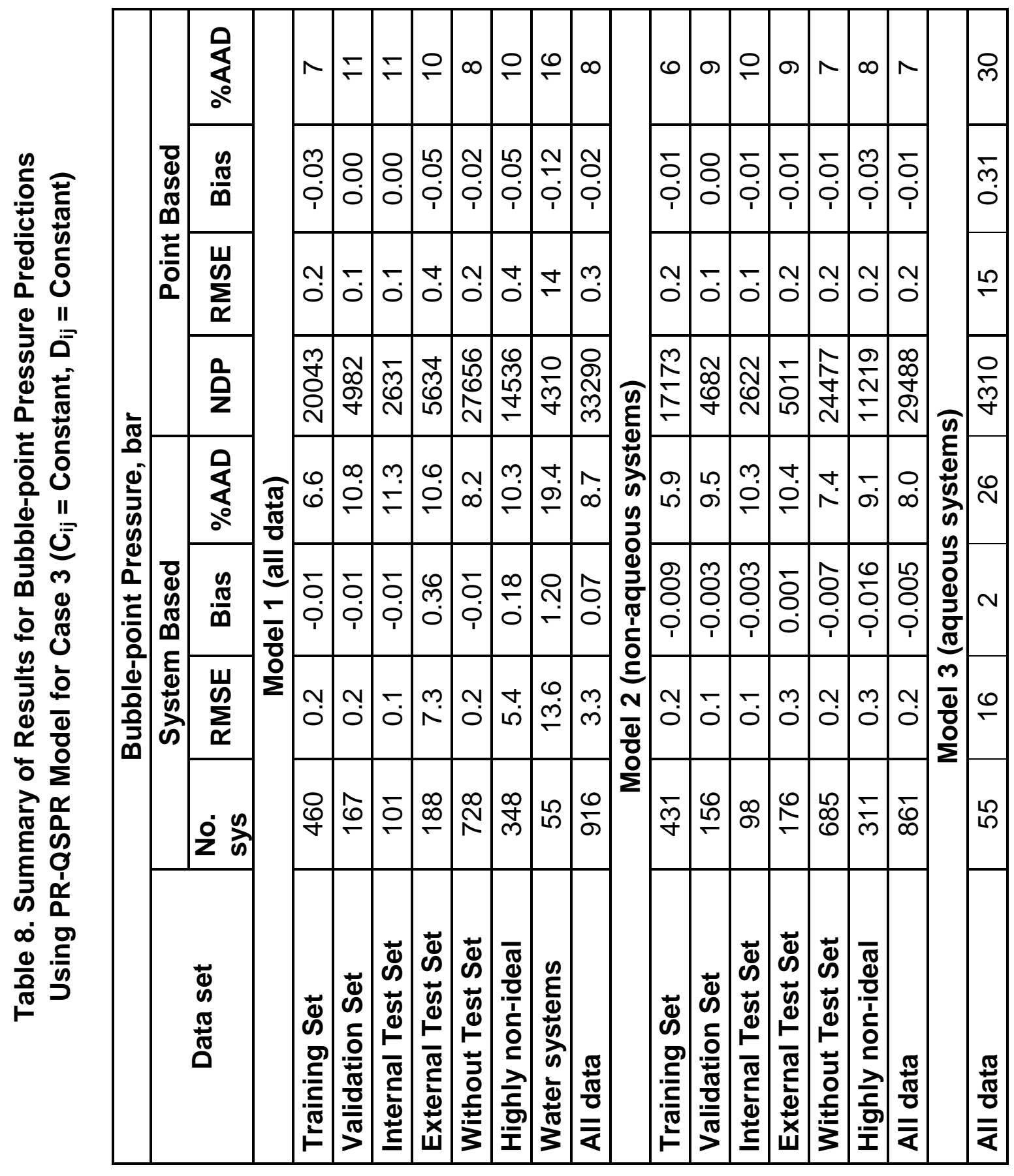




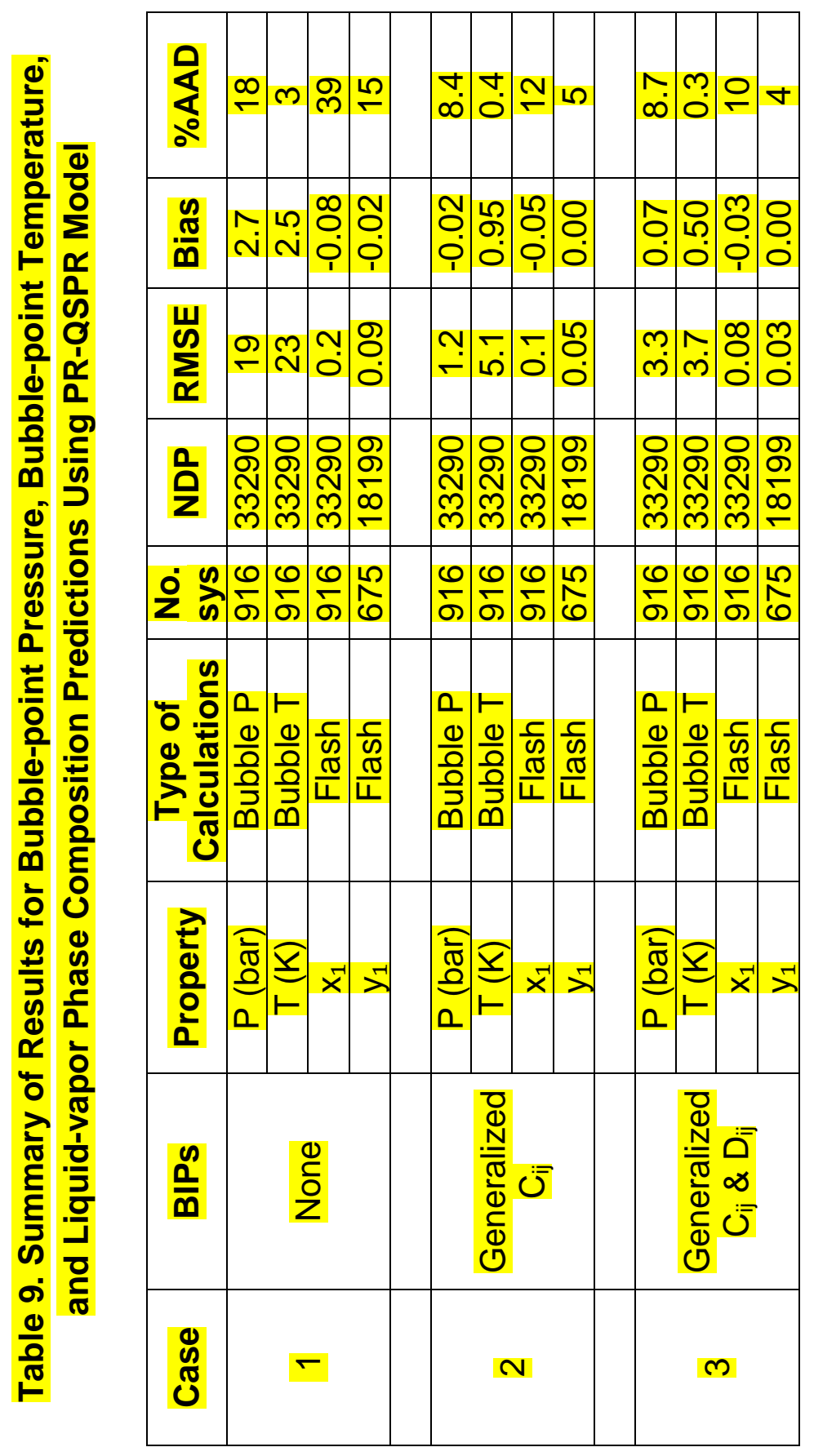




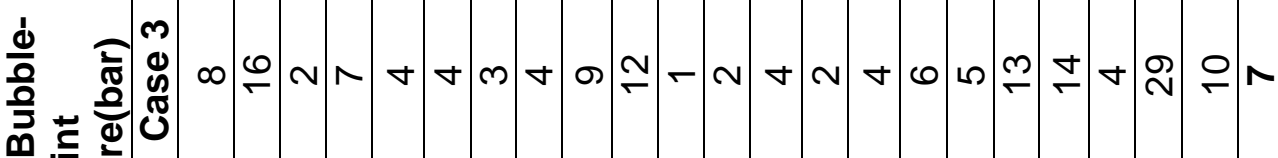

흥

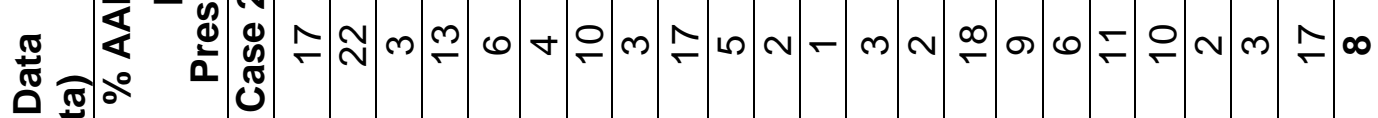

0 0 0

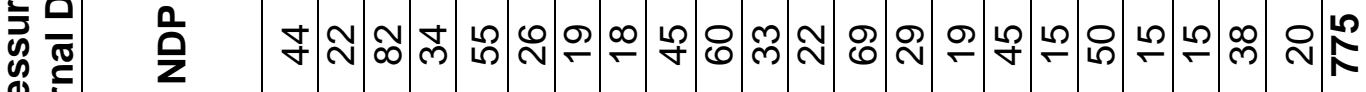

बे

ㅇ क

के ह

N

$\Phi$ N

ณั

(1)

㱐

\& 0

는

댕

응몬

웅호

등ํ

으ㅇㅜㅗ

ติ흥

흥

ต $\overline{0}$

홍응

\&

舫

능 을

즌

สำ

E

ह

ज ช

은

흐원

옳

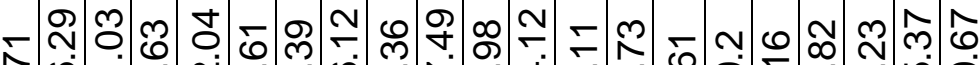

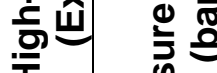

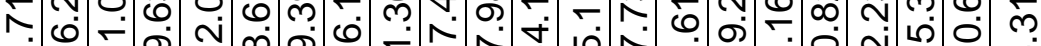

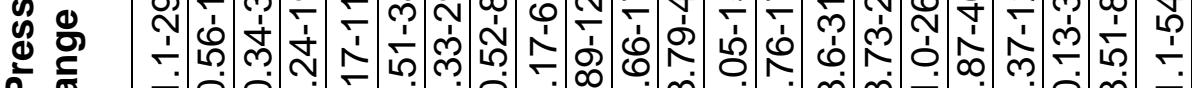

क்

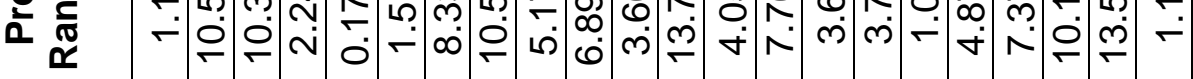

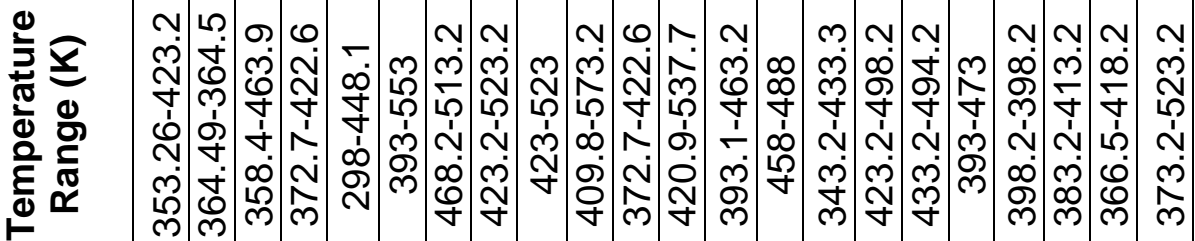

d

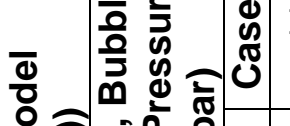

要

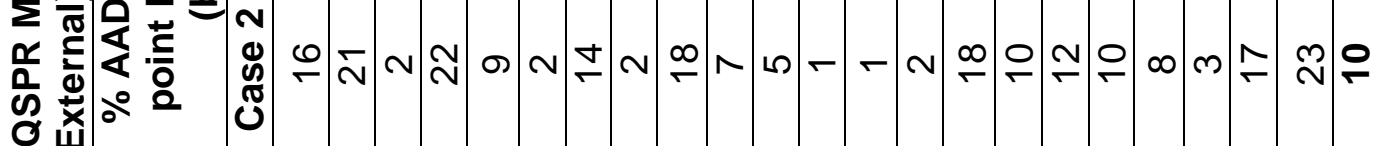

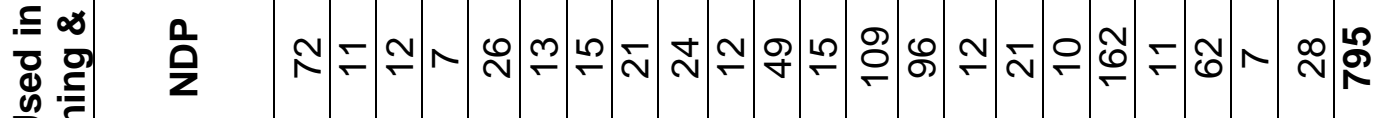

包

吾

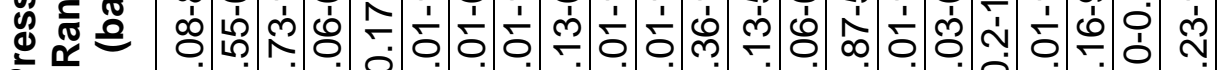

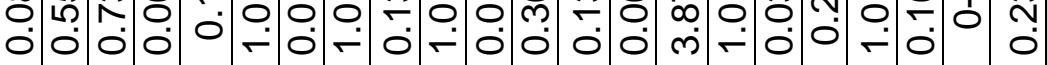

क

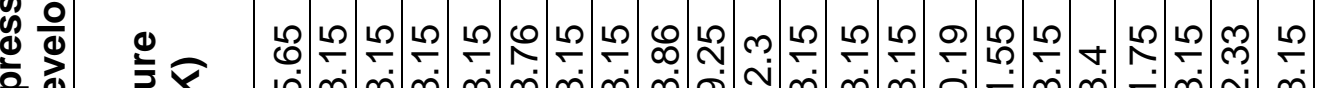

ம்

లి సి ని ని

to

๕

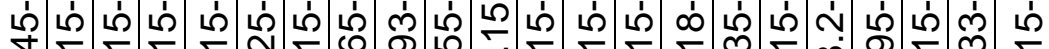

屯 ⿷匚

N N $\mathbb{N}$ N

$\overbrace{\text { के }}^{\frac{E}{\omega}}$

ลิ

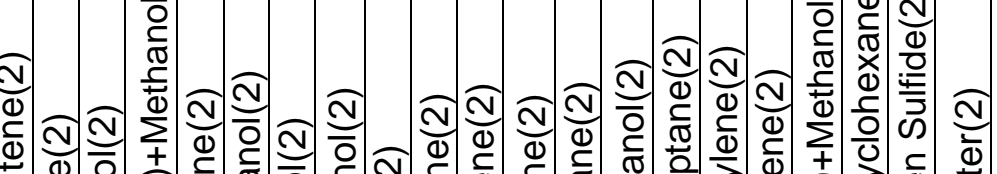

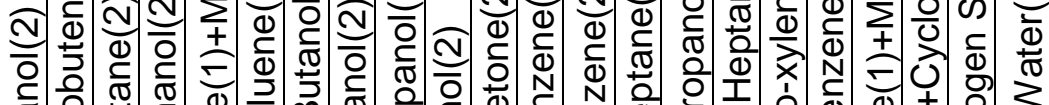

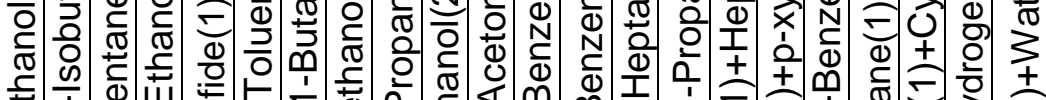

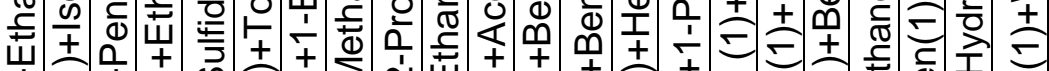

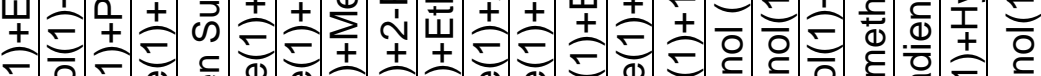




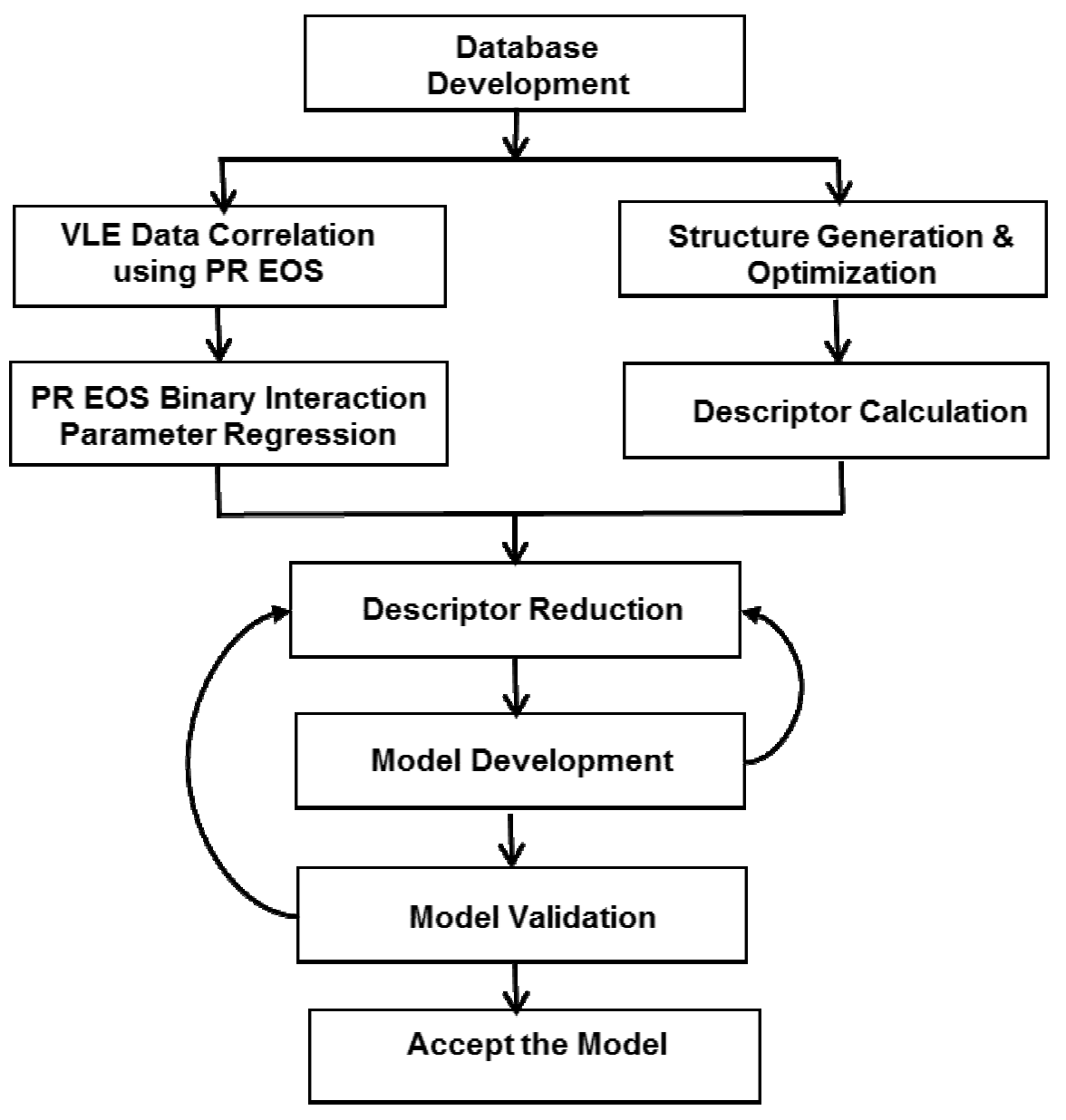

Figure 1. Steps Involved in Development of a QSPR Model 


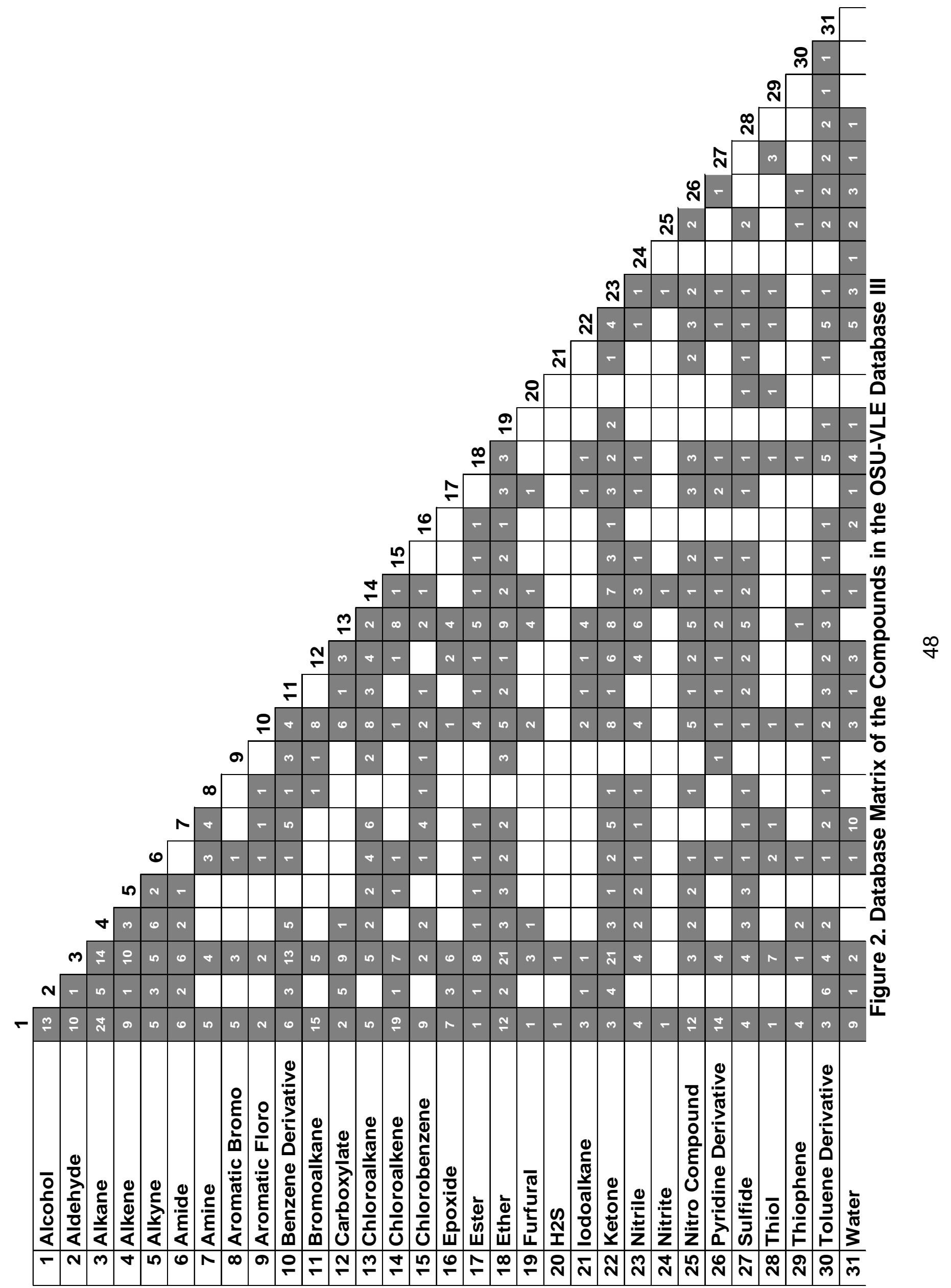




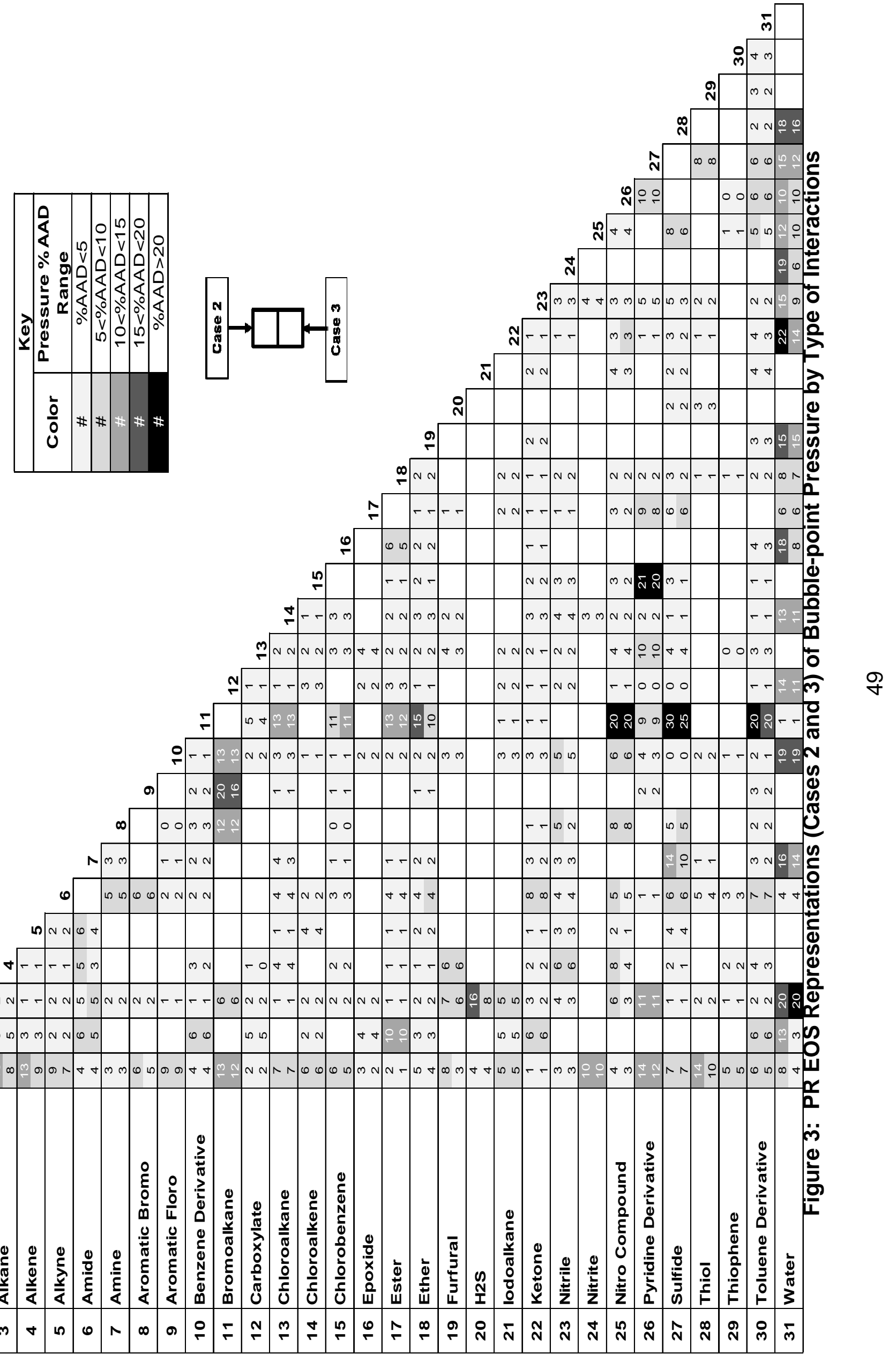




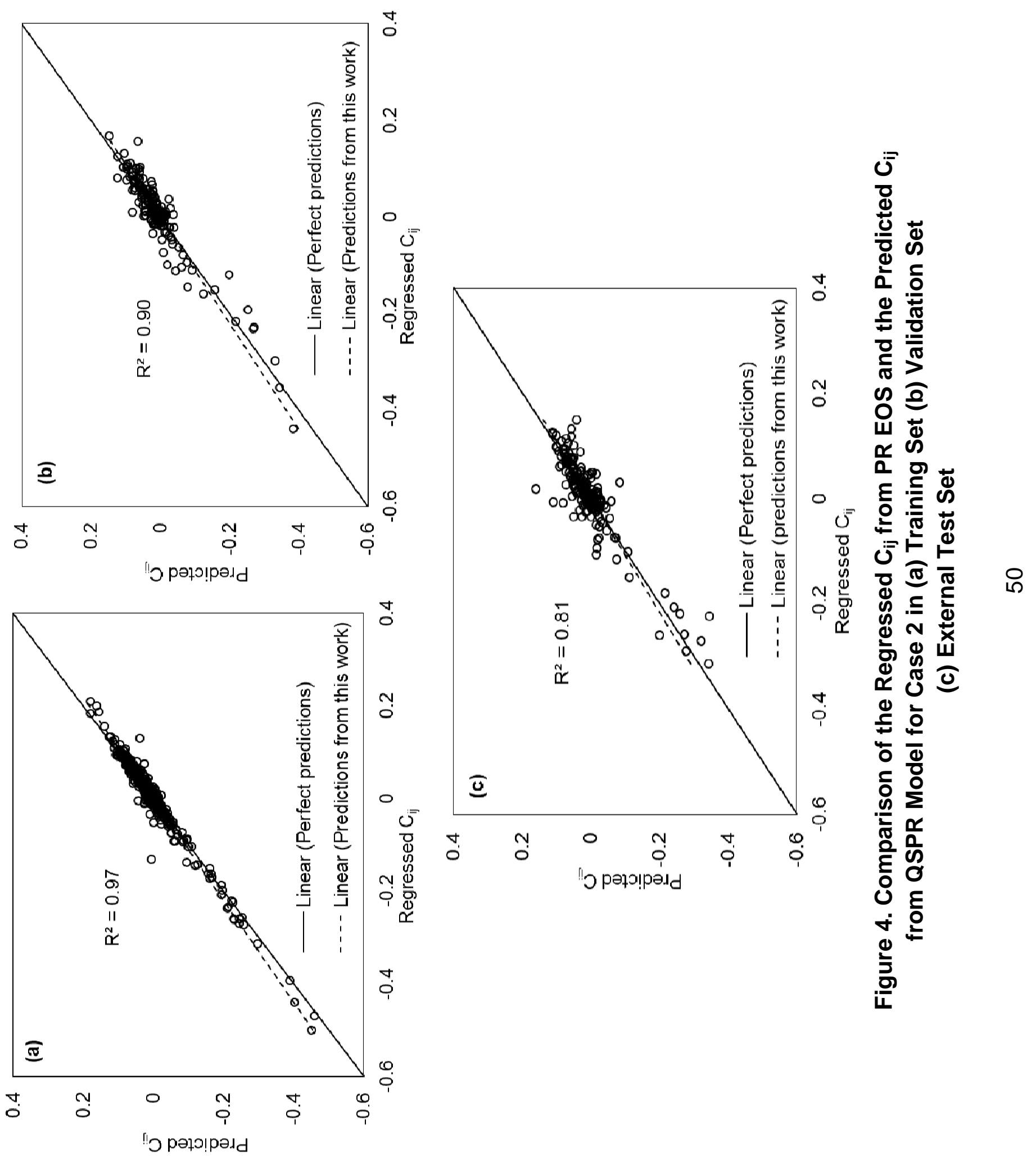




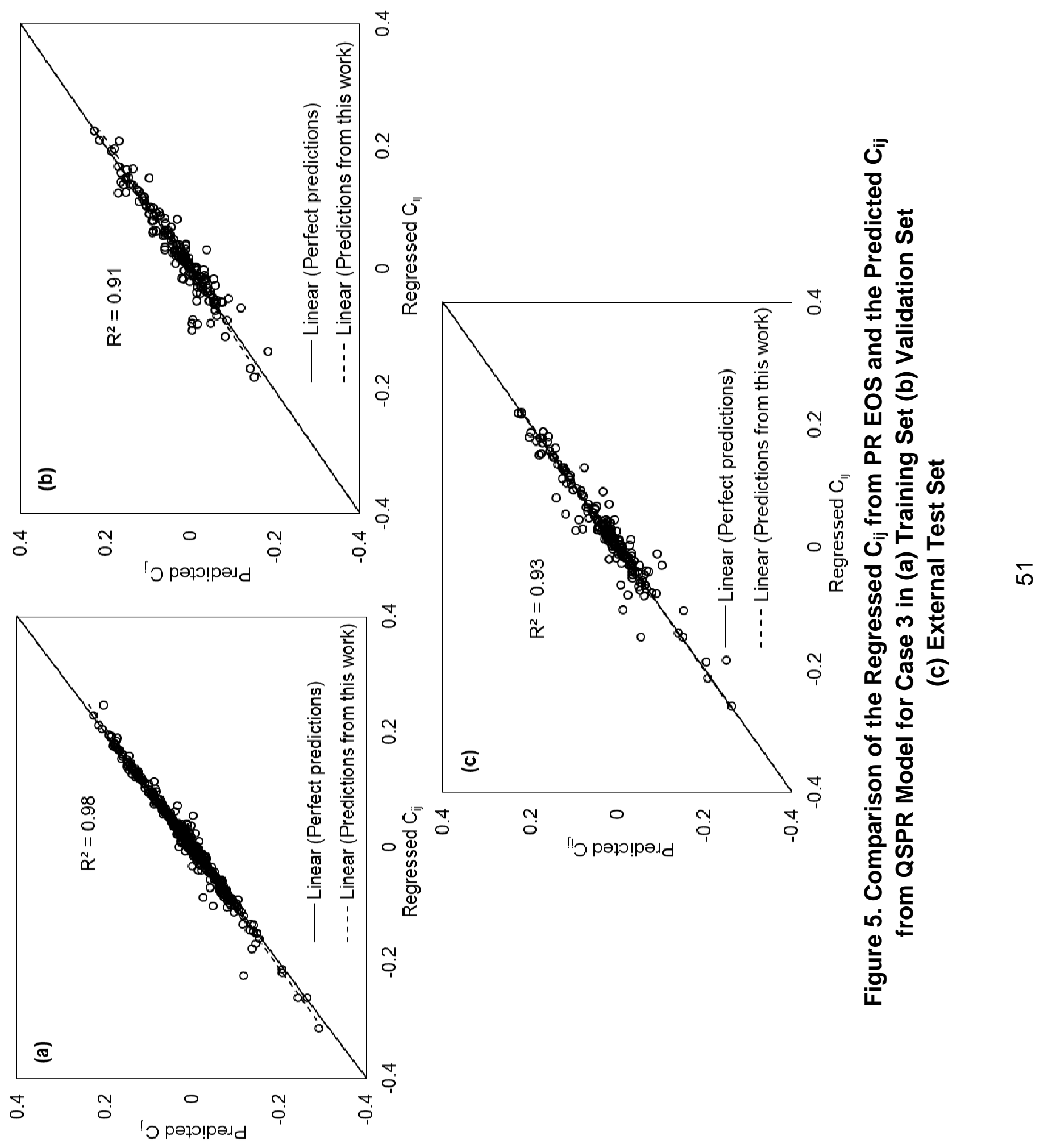



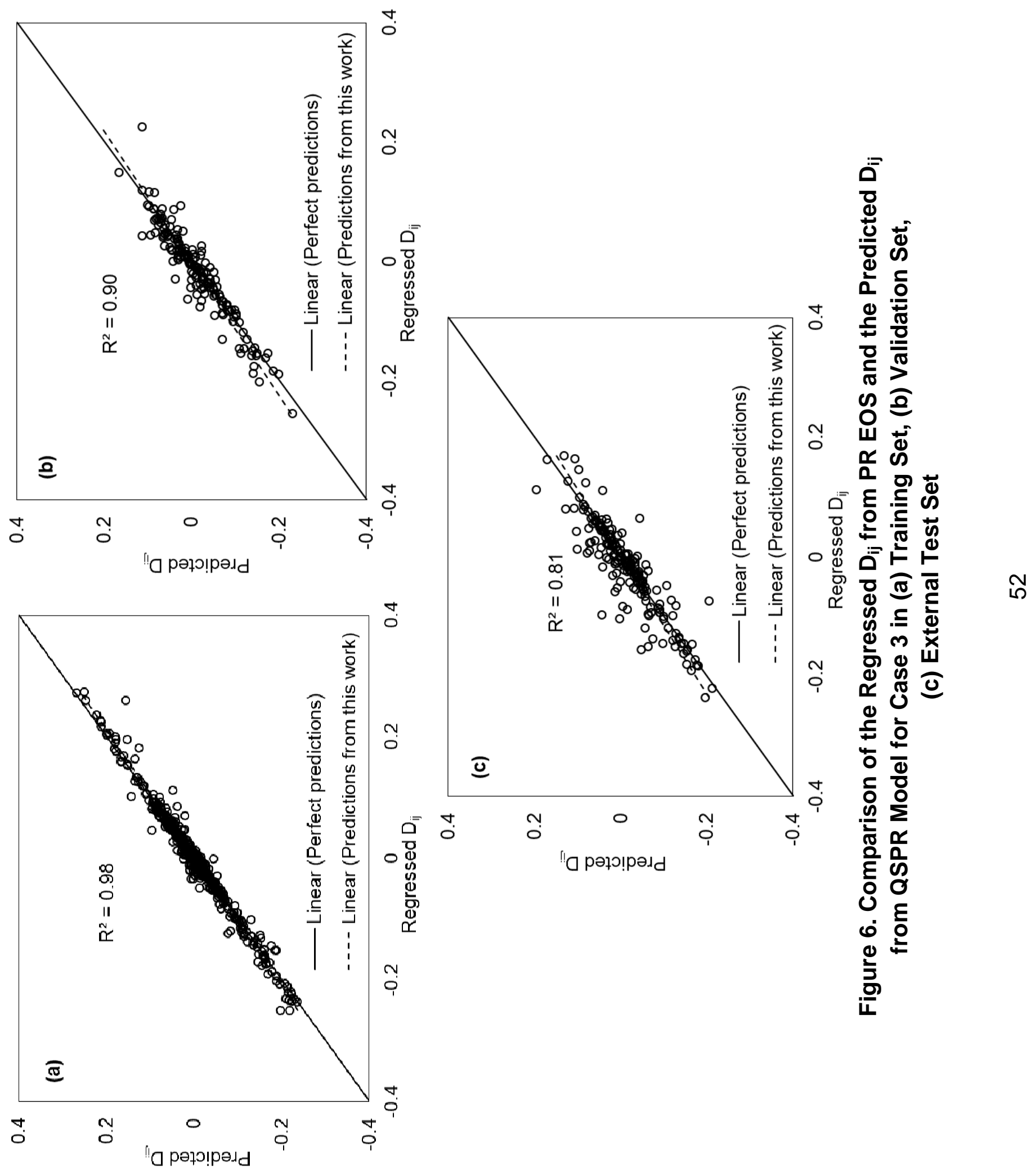


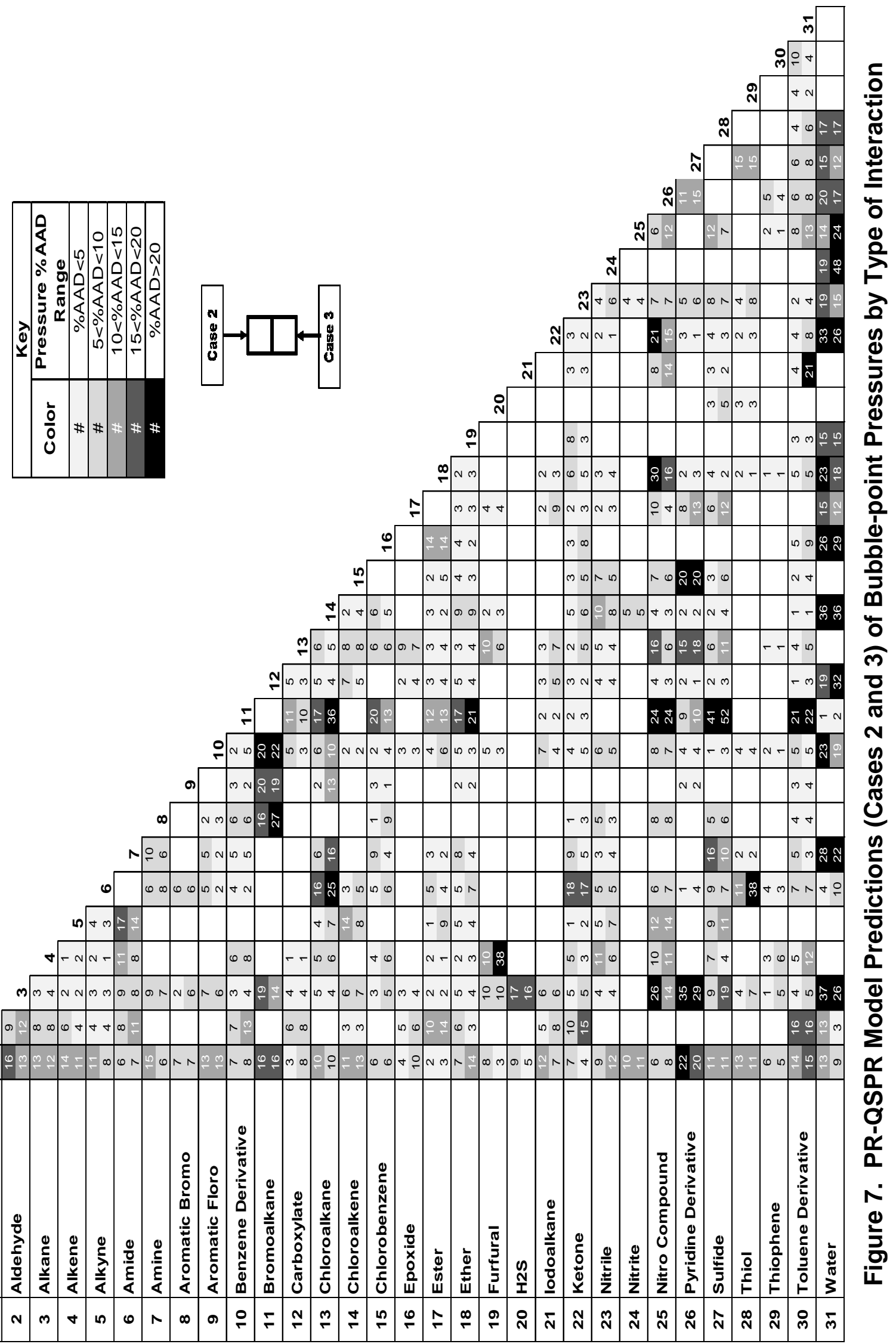

กี 


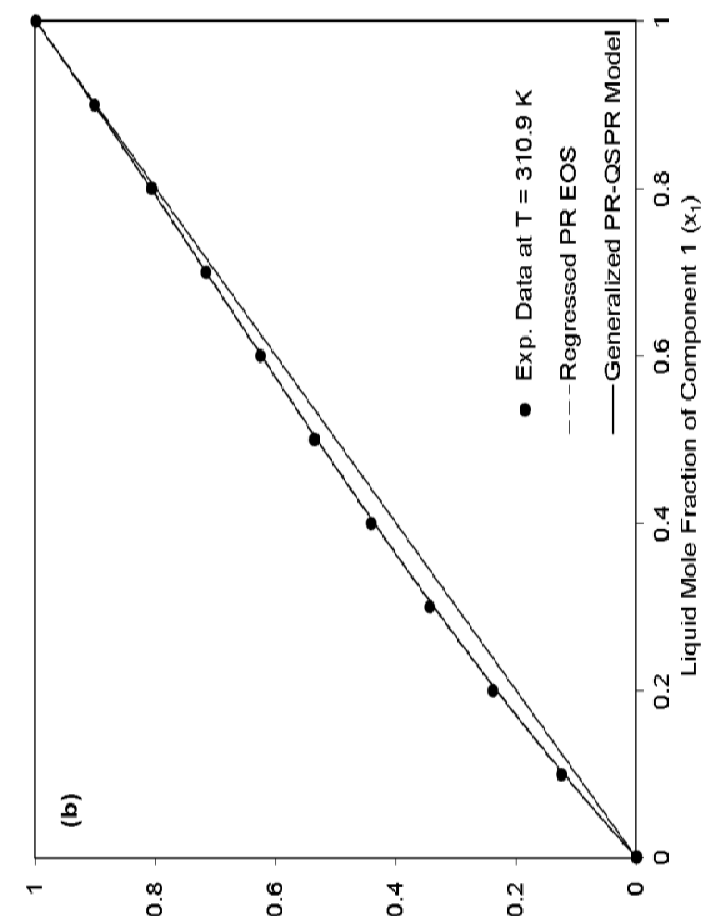

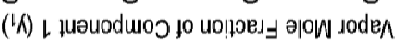

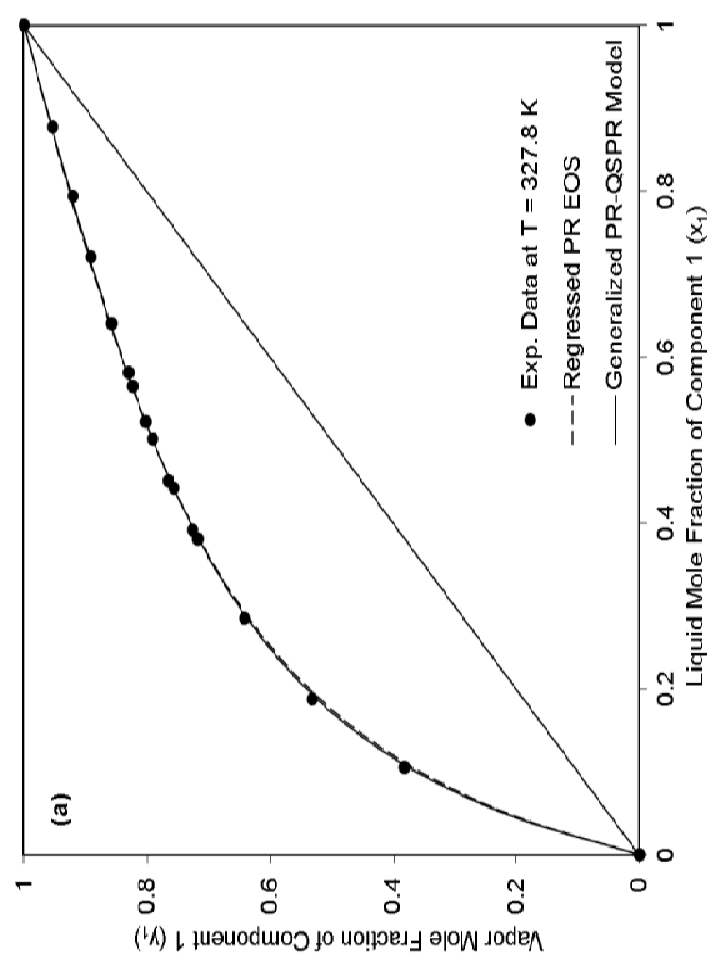

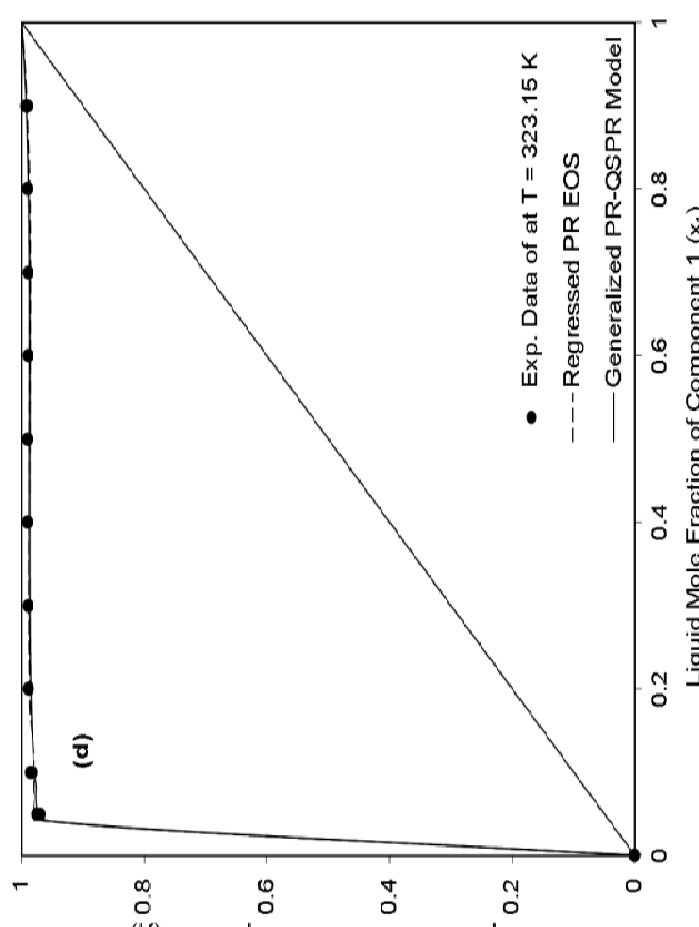

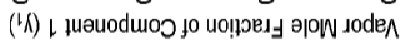

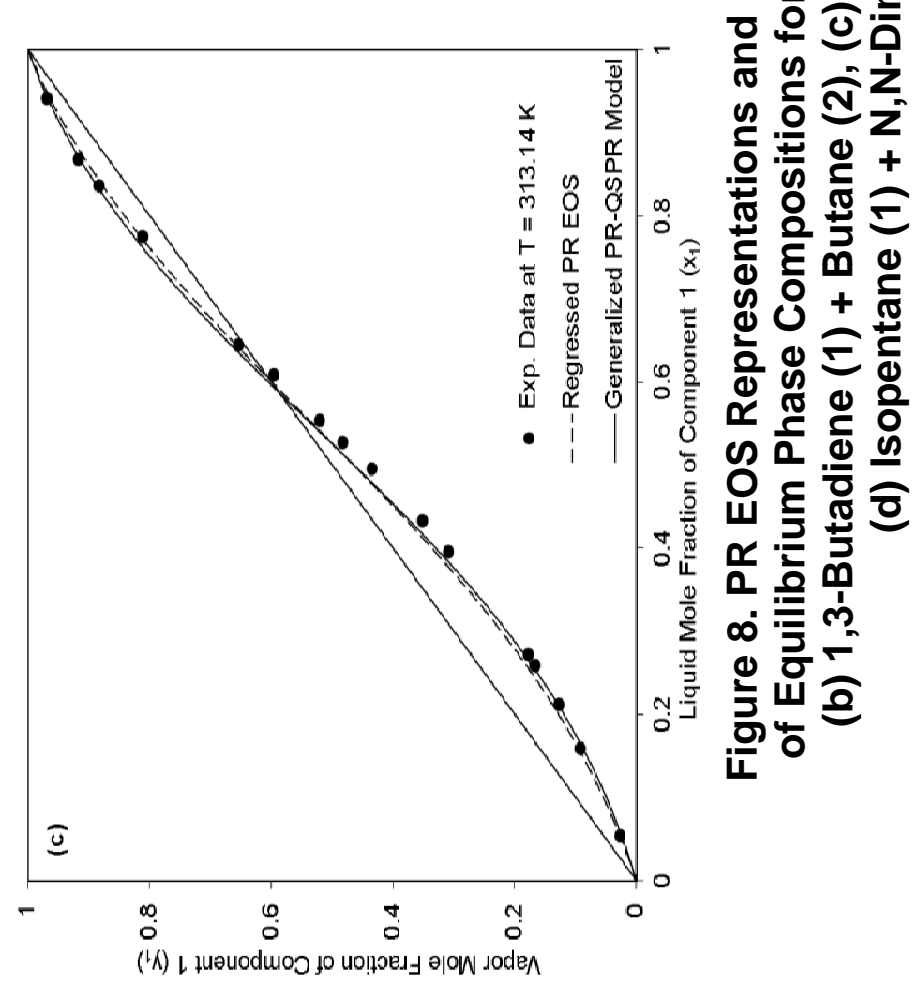



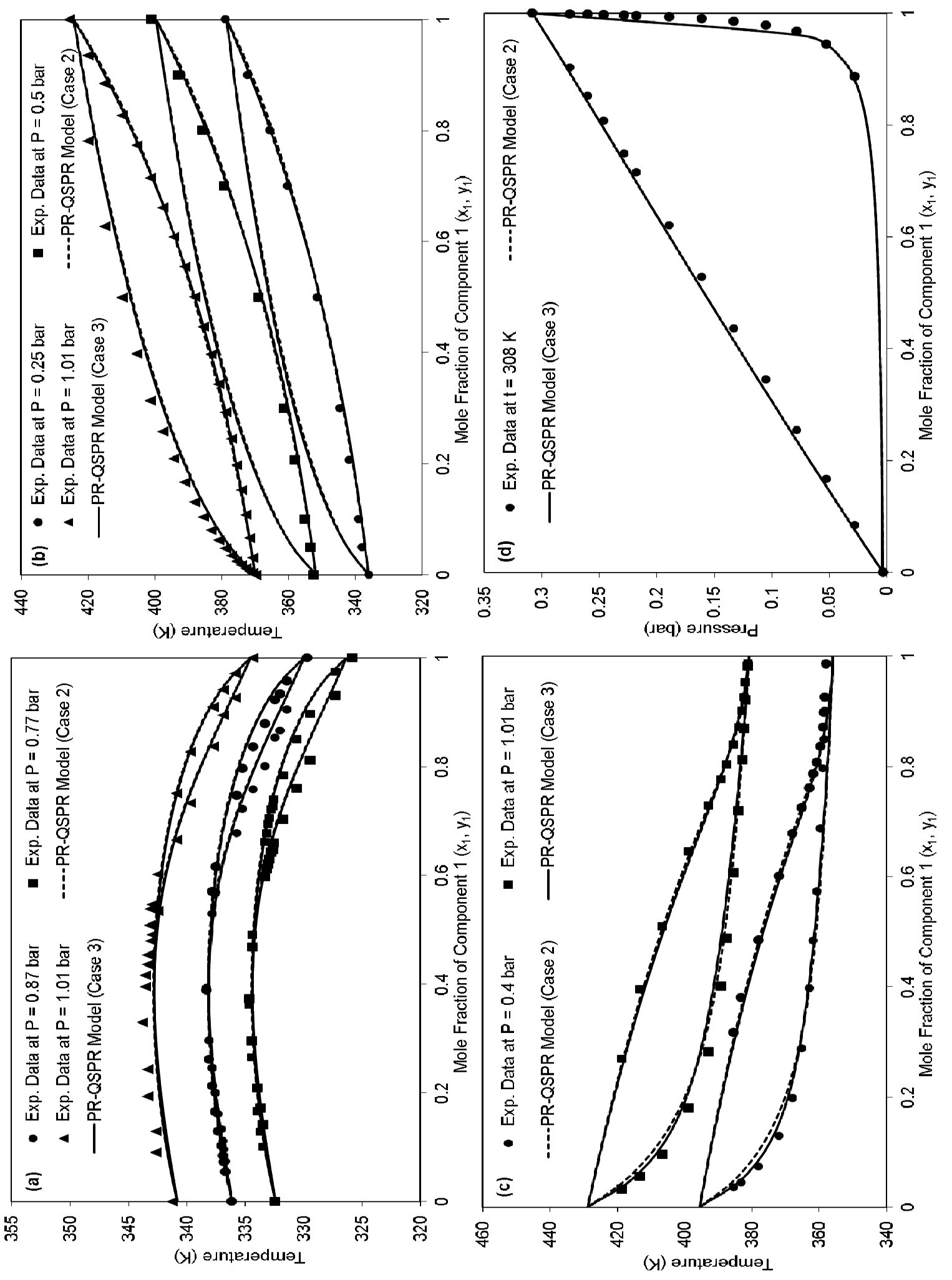

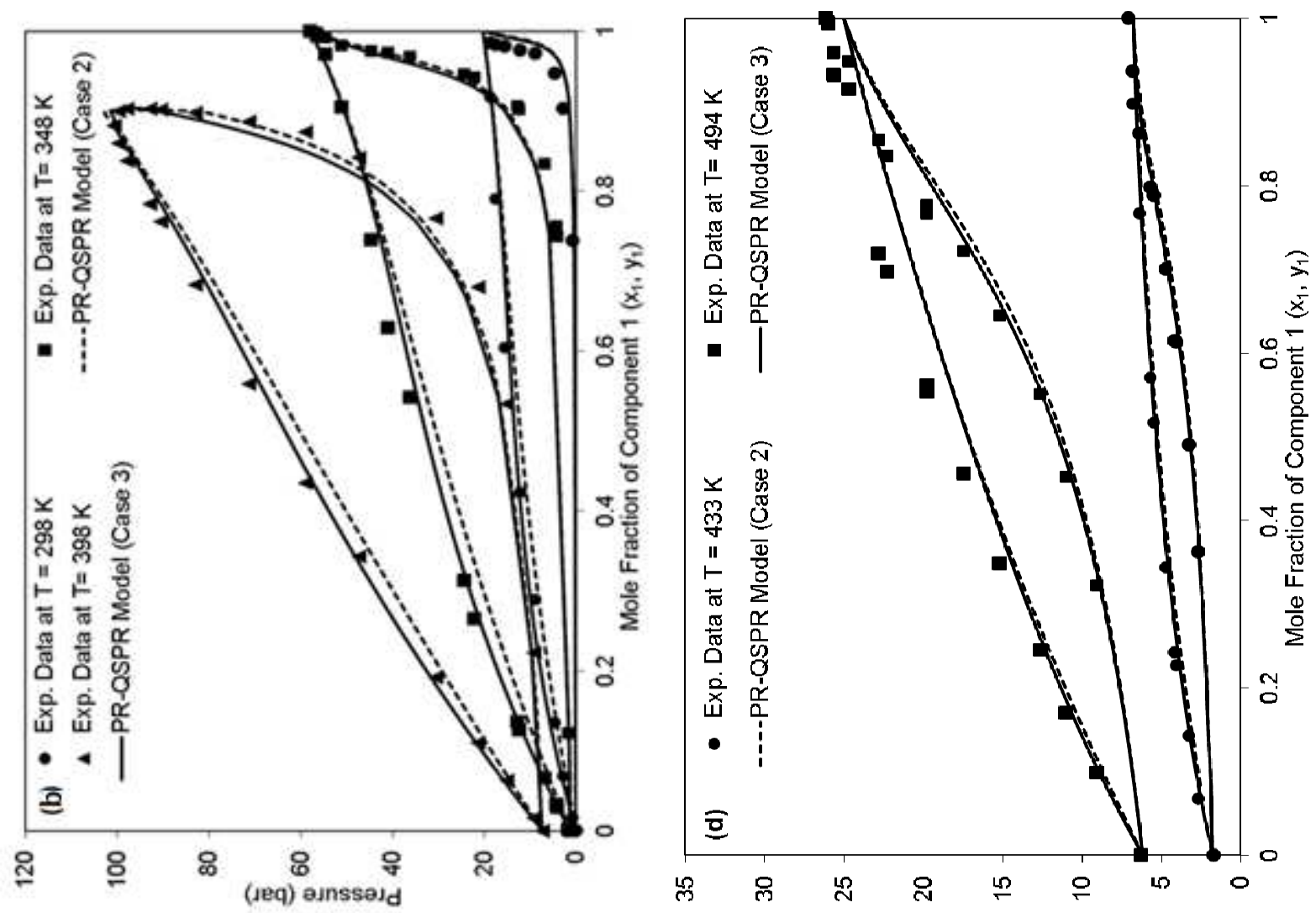

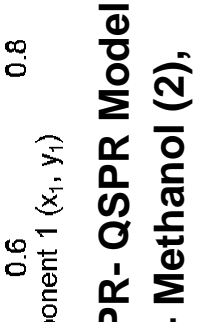

$0+$

$\stackrel{+}{=}$

更

이웡 $\frac{0}{0}$

产 的

¿ 仓

क्ष

는 응

도+

웅 촐

ส

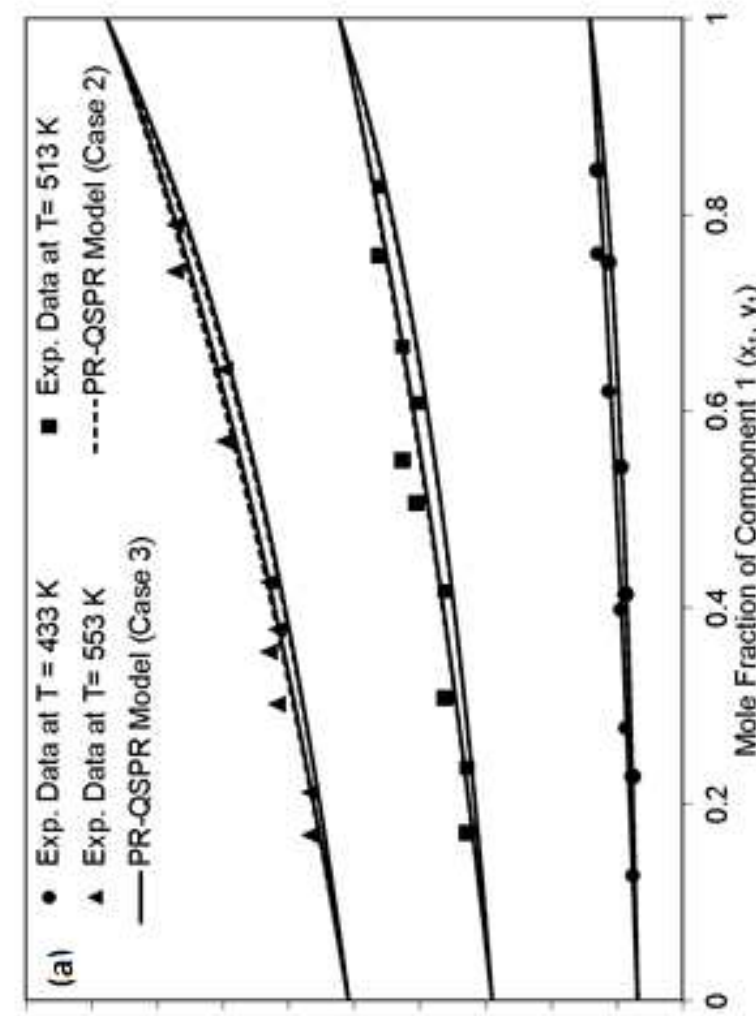

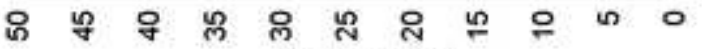

(Jeq) อנnssejd

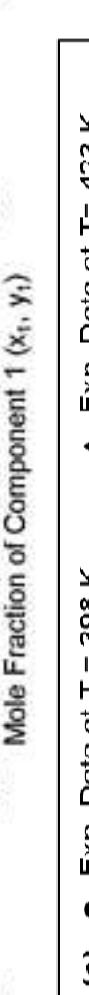

(ueq) əunsse

ह $\frac{1}{\circ}$

들 응

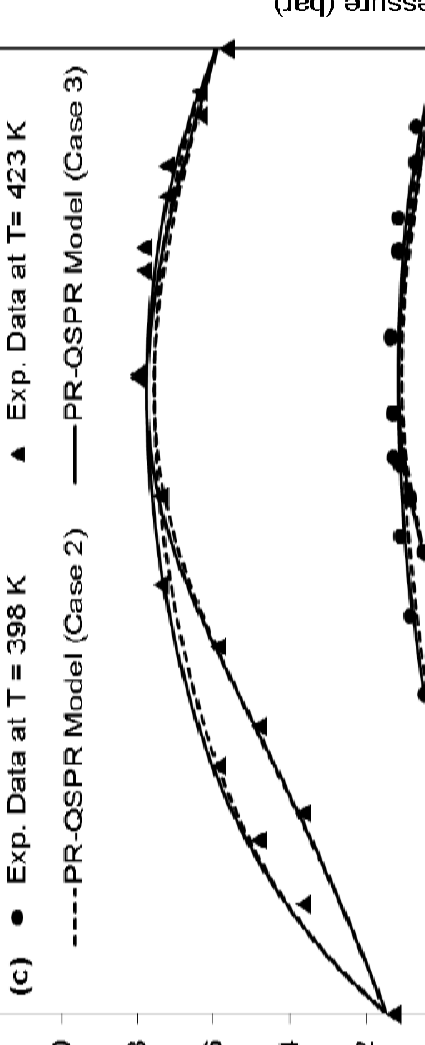

을 무

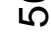

